\title{
Efficient Radio Transmission with Adaptive and Distributed Beamforming for Intelligent WiMAX
}

\author{
Xiaohua Lian · Homayoun Nikookar · Leo P. Ligthart
}

Published online: 2 March 2011

(C) The Author(s) 2011. This article is published with open access at Springerlink.com

\begin{abstract}
In this paper, we introduce Adaptive Beamforming (AB) and Distributed Beamforming (DB) as two efficient techniques for Intelligent WiMAX (I-WiMAX). I-WiMAX is a new maritime communication system, consisting of Smart Radio (SR) principles and mobile WiMAX based on the IEEE 802.16e standard. Adopting AB, the same spectrum can accommodate more users by directing the main beam towards desired users while antenna pattern nulls towards others. To reduce the channel interferences caused by spectrum reusing, the Null Broadening (NB) method is required for downlink beamforming of I-WiMAX. A new $\mathrm{NB}$ method is presented in this paper with iterative weights computing for different OFDM subcarriers. Considering the spatial channel model seen at Subscriber Stations (SS) based on the von Mises distribution, the coverage range of one Base Station (BS) is dramatically enlarged by employing the NB technique. Meanwhile, assuming those SS which are within the coverage range as nodes of a relay network, the DB technique is introduced to forward signals to distant SS by forming the main beams towards them instead of deploying more BS to increase the communication range. It is proven in this paper that DB can successfully do the job. To reuse the spectrum, two DB methods are presented by directing different beams towards different required directions at the same time. With the $\mathrm{AB}$ and $\mathrm{DB}$ techniques, reliable I-WiMAX links can be established for long distances, with more optimum spectrum use and less infrastructure.
\end{abstract}

Keywords Intelligent WiMAX - Smart radio - Adaptive beamforming - Distributed beamforming $\cdot$ Collaborative beamforming $\cdot$ Smart antenna $\cdot$ Adaptive OFDM

\footnotetext{
X. Lian $(\varangle) \cdot$ H. Nikookar · L. P. Ligthart Department of Electrical Engineering, Mathematics and Computer Science, International Research Center for Telecommunications and Radar (IRCTR), Delft University of Technology, Mekelweg 4, 2628 CD Delft, The Netherlands e-mail: x.lian@tudelft.nl

H. Nikookar e-mail: h.nikookar@tudelft.nl

L. P. Ligthart e-mail: 1.p.ligthart@tudelft.nl
} 


$\begin{array}{ll}\text { Abbreviations } \\ \text { AB } & \text { Adaptive Beamforming } \\ \text { AOFDM } & \text { Adaptive Orthogonal Frequency Division Multiplexed } \\ \text { AOA } & \text { Angle of Arrival } \\ \text { BS } & \text { Base Station } \\ \text { CDF } & \text { Cumulative Distributed Function } \\ \text { CMA } & \text { Constant Modulus Adaptive } \\ \text { DB } & \text { Distributed Beamforming } \\ \text { DOA } & \text { Direction of Arrival } \\ \text { DSS } & \text { Distant SS } \\ \text { FDD } & \text { Frequency Division Duplex } \\ \text { GPS } & \text { Global Positioning System } \\ \text { I-WiMAX } & \text { Intelligent WiMAX } \\ \text { LMS } & \text { Least Mean Square } \\ \text { MLM } & \text { Maximum Likelihood Method } \\ \text { MMSE } & \text { Minimum Mean Square Error } \\ \text { MS } & \text { Mobile Station } \\ \text { MVDR } & \text { Minimum Variance Distortion Response } \\ \text { NB } & \text { Null Broadening } \\ \text { NC } & \text { Null Constraint } \\ \text { OFDM } & \text { Orthogonal Frequency Division Multiplexed } \\ \text { Pdf } & \text { Probability density function } \\ \text { SA } & \text { Smart Antenna } \\ \text { SCORE } & \text { Spectrum self-COherent REstoral } \\ \text { SDMA } & \text { Space Division Multiple Access } \\ \text { SINR } & \text { Signal to Interference plus Noise Ratio } \\ \text { SIR } & \text { Signal to Interference Ratio } \\ \text { SMI } & \text { Sample Matrix Inversion } \\ \text { SNR } & \text { Signal to Noise Ratio } \\ \text { SR } & \text { Smart Radio } \\ \text { SS } & \text { Subscriber Station } \\ \text { TDD } & \text { Time Division Duplex } \\ \text { ULA } & \text { Uniform Linear Array } \\ \text { VDA } & \text { Virtual Direction Adding } \\ & \end{array}$

\section{Introduction}

Beamforming techniques have been widely introduced to many wireless communication systems to improve the system Signal-to-Noise-Ratio (SNR), which will consequently increase system data rates and enlarge the coverage range. I-WiMAX has been introduced as a new maritime communication system which combines Smart Radio (SR) and Mobile WiMAX in an intelligent way [1]. This paper presents the applications of two different beamforming techniques to I-WiMAX: Adaptive Beamforming ( $\mathrm{AB}$ ) and Distributed Beamforming (DB).

\subsection{I-WiMAX: A New Maritime Communication System}

I-WiMAX is aimed to provide a metropolitan access network, and should offer higher bandwidth, larger coverage in maritime wireless communications compared to existing wireless 


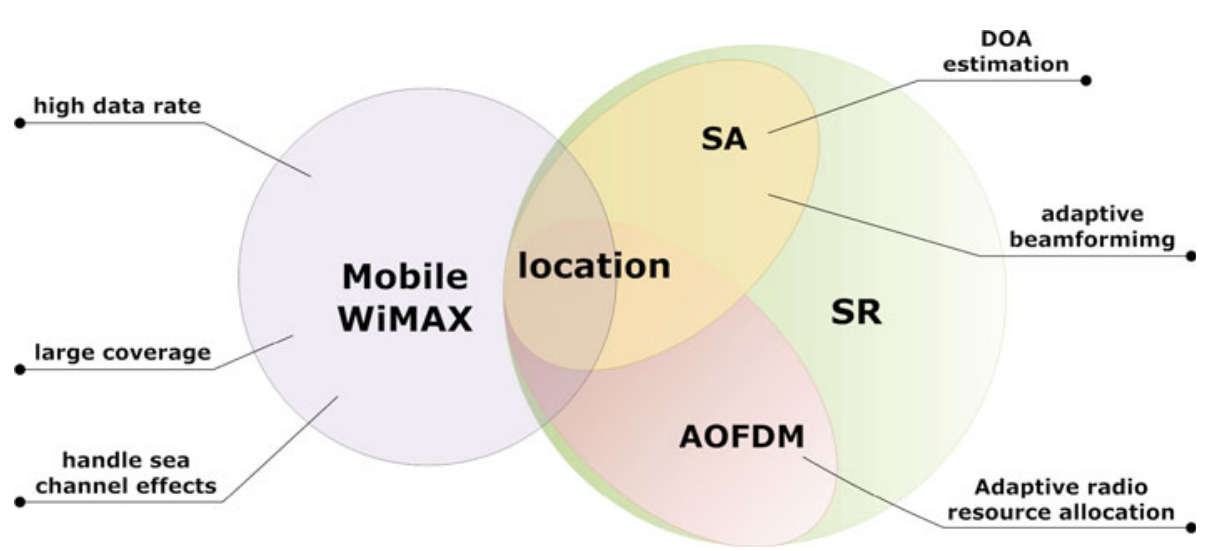

Fig. 1 I-WiMAX concept of maritime coastal/lake communications and locationing

systems. It is built upon SR and mobile WiMAX, as shown in Fig. 1 [1]. SR is a crucial part embedded in I-WiMAX to be applied into maritime communications. With SR, the new communication system gains knowledge on the severe sea communication environment, full of echoes and reflections, adapts to it, and therefore increases its cognition and flexibility. The SR technique, which is adopted on top of WiMAX, basically contains two techniques, Adaptive OFDM (AOFDM) and Smart Antenna (SA). AOFDM adaptively allocates the radio resource and consequently guarantees the reliable link in the sea channel full of fading and reflections caused by the rough sea surface. SA ensures a higher SNR which consequently results into larger coverage areas by performing adaptive beamforming.

With I-WiMAX, the people on board of ships are capable of enjoying a large amount of information anywhere and anytime. Therefore the transportation efficiency will be significantly improved by the abundant and versatile information obtained from the new maritime communication system based on broadband wireless networks. Furthermore the locationing ability of the broadband communication system is integrated, offering position services via RF localization.

\subsection{Pervasive Adaptive Beamforming Techniques for Wireless Communications}

SA is an effective technique for every energy-efficient radio because it can, in the transmit mode, focus the energy in the required direction and assist in reducing the multipath reflections and delay spread [2], caused by the fact that a desired signal can arrive via different directions [3]. In the receive mode, it can also perform optimal combining after delay compensation of the incoming multipath signals [4]. According to [5], an $M$-element adaptive array and a multi-beam antenna provide an $M$-fold increase in antenna gain. This increases the range and reduces the number of base stations required to cover a given area. An adaptive beamformer for the uplink WiMAX compliant system was proposed in [6], which has demonstrated its ability to cope with time varying interference effectively. For WiFi in maritime scenarios, it has been shown in [6] that if a sectorized antenna is employed instead of an omni-directional antenna, the distance tested in a boat-to-air experiment can be at least doubled.

Recently an experimental adaptive beamforming system for the IEEE802.16e-2005 OFDMA downlink has been implemented by Motorola labs [7,8]. This system consists 


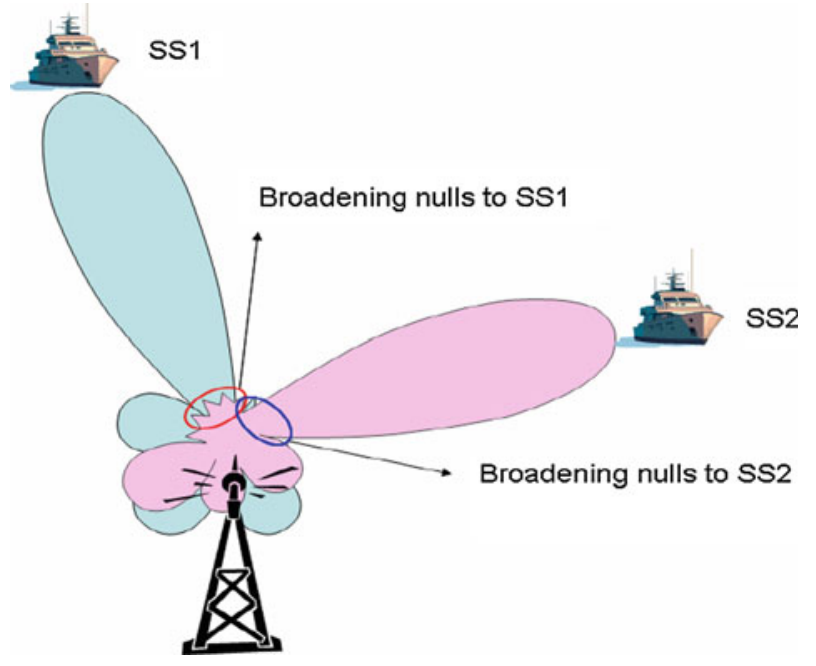

Fig. 2 Null broadening technique for an adaptive OFDM beamformer for I-WiMAX (SS1 and SS2 share the same OFDM subcarriers)

of a real time-division duplex link between the Base Station (BS) array antenna and a single antenna Subscriber Station (SS). The test results show that the Mobile WiMAX is capable of obtaining significant coherent processing gains allowed by array antenna channels. The requirements for supporting SA by WiMAX are discussed in detail in [9], as well as the protocol support and the complexity of extra signal processing as a result of beamforming.

In this paper, we propose an efficient spectrum usage of green radio, which means that all SS are assigned to all OFDM sub-carriers. Since we serve more than one user on a single traffic channel, co-channel interference suppression is our goal to achieve. Due to the spatial filtering function of SA, co-channel interferences may be reduced at both transmit and receive side by forming beams with nulls in the direction of those SS who share the same spectrum band [10-12]. As a result, it may lead to an increased number of users [13,14], which consequently improves the channel capacity and spectrum efficiency [15].

An uplink adaptive beamforming technique can direct its main beam towards the interested signal source, while displaying nulls to the unwanted directions. This ensures that the transmitted signal from one of the SS can be detected and selected by the BS via beamforming. Therefore, for those re-used OFDM sub-carriers, the weights should guarantee the main beam directed towards the desired SS, while at the same time "null" beams are created towards other SS.

For downlink, since SS does not have SA, appropriate beamforming techniques must be provided by the BS to satisfy the link quality for all co-channel SS. If we only use the estimated weights of uplink for the downlink, the nulls in the patterns towards those co-channel SS may become too narrow. Therefore, Null Broadening (NB) methods for beamforming should be considered [16]. Employing the NB technique, the adaptive downlink beamforming of I-WiMAX for green radio is shown in Fig. 2. Unlike the point nulling technique, the downlink beamforming with null broadening will spread the nulling over an angular region around a certain direction. I-WiMAX performs downlink beamforming with depressed power in a certain angular range to guarantee that the co-channel interference caused by spectrum reusing is reduced for SS. 


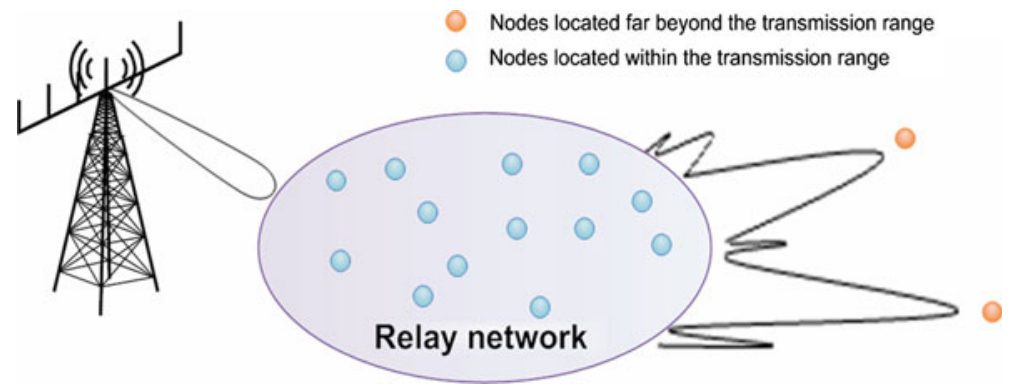

Fig. 3 Distributed beamforming for I-WiMAX in long distance communications

In this paper, we present a new NB method. It can be regarded simply as adding the coherent signals to each available signal source, but operating in two different directions; therefore it is called Virtual Direction Adding (VDA). Meanwhile, we also give a closed-form relationship between the weights of the Minimum Variance Distortionless Response (MVDR) beamformer and those with broadened nulls. Based on this result, we show that the weights of downlink beamforming with NB can be calculated iteratively. In addition, we demonstrate and compare VDA with another NB method, and show that when considering the spatial channel seen at the SS, the proposed NB method for downlink beamforming is highly required and it decreases co-channel interferences significantly.

\subsection{Distributed Beamforming: A New Concept Beamforming Technique}

In the case when some of the SS are beyond the transmitting range of each SS, we propose a solution to build a long distance energy-efficient link for I-WiMAX, as demonstrated in Fig. 3. We suggest the "close" SS, which are located within the transmission range, to form a relay network forwarding BS signals by employing a beamforming technique to "far" SS, which are beyond the transmission range. The BS is equipped with SA, and then it can steer the main beam towards the direction of the relay network. We assume that the main beam is wide enough, and then all the nodes share the same information simultaneously. All relay nodes will constructively display a spatial beam pattern, directing mains beams towards those "far" SS. With the energy focused only in these directions, signals can be transmitted properly to a distant SS. Consequently, the BS cell coverage can be enlarged significantly without needing extra infrastructure.

The difficulty of the beamforming shown in Fig. 3 is that the positions of SS in the relay network for I-WiMAX are random. Unlike fixed antenna array elements, each SS can appear anywhere within the coverage range. Therefore, another beamforming technique (which is based on distributed array antenna elements), rather than $\mathrm{AB}$ should be considered.

DB is originally employed as an energy-efficient scheme to solve the long distance transmission by wireless sensor networks, in order to reduce the amount of energy required and consequently to extend the utilization time of the sensors. The basic idea of DB is that a set of nodes in wireless networks are treated as a virtual antenna array and then form a beam towards a certain direction to collaboratively transmit a signal. The direction of the main beam can be adjusted by setting the initial phase of each node. It is also regarded as collaborative beamforming. 
Based on the work in [17], DB is proposed in [18] as a new concept of beamforming. By employing $N$ collaborative nodes, the collaborative beamforming can result in up to $N$-fold gain in the received power at a distance access point [18]. A cross-layer approach to DB for wireless ad-hoc networks is discussed in [19] with more complicated models and two time phases of communication steps. The improved beampattern and connectivity properties are shown in [20], and a reasonable beamforming performance with node synchronization error is discussed in [21]. DB requires accurate synchronization; in other words, the sources must transmit at the same time, synchronize their carrier frequencies and control their carrier phases so that their signals can be combined constructively at the destination. Several synchronization techniques for DB can be found in [22,23].

As shown in the model of Fig. 3, by introducing DB to the relay network, not only BS increases its transmission range, but also each SS as a collaborative relay node can reduce its transmission power to preserve its energy. As mentioned before, we propose that with the adaptive beamforming technique, the BS can transmit signals by steering the main beam towards one SS and nulls towards others while efficiently using the spectrum. This basic idea can also be implemented in DB for the relay network. It requires for the nodes of the relay network to form a beampattern with different main beams towards different directions of distant SS. If we adopt the initial phases proposed for each node as in [18], only a single main beam towards one direction can be generated at one time. Therefore, we need to separately form different main beams in divided time slots, which is not time-efficient. Meanwhile, synchronizing the initial phases of nodes in each sub-time slots will also be required.

In this paper, we present two DB methods that can form multi-beams. The first method is to separate the nodes geographically into several ring ranges and a smaller circular range, while nodes at different ranges adopt different initial phases. Then by combining them together constructively, we can have beams towards several required directions. Another technique is to assign initial phases to each node randomly. Both DB methods can display multi-beam towards different destinations successfully, and only require one time of synchronization, instead of nodes synchronized each time when the beam direction changes. The Cumulative Distributed Function (CDF) of the beampattern, when the number of nodes is large, is discussed, showing the probability of a certain amount of energy that can be received at different directions.

\subsection{Organization of the Paper}

This paper is organized as follows. First in Sect. 2, we discuss AB for I-WiMAX. This includes the uplink and downlink Adaptive Beamformers. Meanwhile, a new NB method as a co-channel interference reduction technique is shown and demonstrated. In Sect. 3, DB for I-WiMAX is explained. We show that with two multi-beam generating DB methods, I-WiMAX is able to achieve communications to SS which are far beyond the transmission range. Finally in Sect. 4, we conclude the paper.

\section{Adaptive Beamforming Technique for I-WiMAX}

In this section we demonstrate that the adaptive beamformers for I-WiMAX allow for efficient spectrum re-using. Both uplink and downlink adaptive beamformers are discussed. Particularly, a new NB method is presented. 


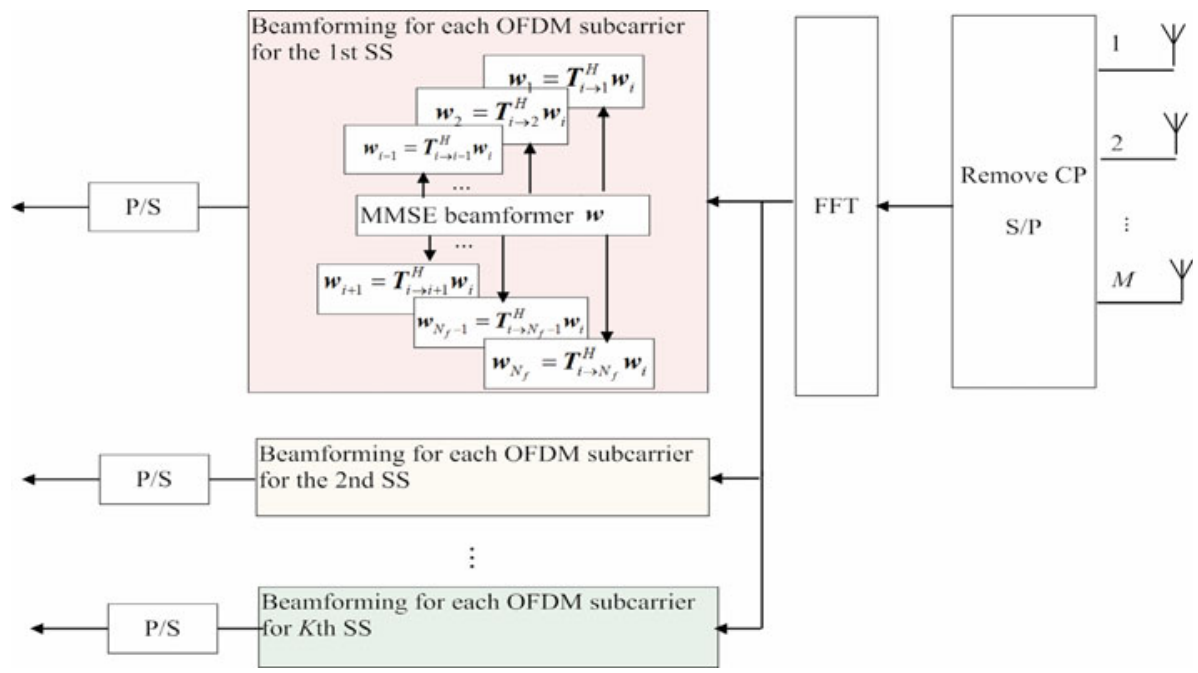

Fig. 4 Adaptive uplink OFDM beamformer of I-WiMAX (P/S: Parallel to Serial; FFT: Fast Fourier Transform; CP: Cyclic Prefix; S/P: Serial to Parallel; $N_{f}$ : number of OFDM subcarriers)

\subsection{Uplink and Downlink Adaptive Beamforming for I-WiMAX}

The uplink adaptive OFDM beamformer of I-WiMAX is illustrated in Fig. 4. The Minimum Mean Square Error (MMSE) beamformer is situated after the FFT for receiving. This is why it is called the post-IFFT/FFT beamformer. Its basic idea is to regard OFDM signals as a combination of several narrowband signals, so that the weights of the MMSE beamformer are decided after the Multi-carrier Modulator/Demodulator (IFFT/FFT) for each OFDM subcarrier. The weights for the OFDM adaptive beamformer are calculated iteratively. As shown in Fig. 4, if one set of weight $\mathbf{w}_{i}$ for the $i$ th OFDM subcarrier is computed, others will be deduced by mapping them into different frequency bands via transformation matrix $\boldsymbol{T}$. Thus the beamforming calculation will be performed only $K$ times for all $K \mathrm{SS}$, which requires less computation load and consumes less time [24].

Several beamforming approaches exist with a varying degree of complexity. The beamforming technique was initially developed in the 1960s for military applications in sonar and radar, in order to remove unwanted noise and jamming from the array output. The first fully adaptive array was conceived in 1965 by Applebaum in [25,26], which was designed to maximize the SNR at the array's output. The Least Mean Square (LMS) error algorithm proposed by Widrow in [27] was regarded as an alternative approach to cancelling the unwanted interferences. Further work about LMS techniques was done by Frost in [28] and Griffiths in [29] by introducing the constraints to ensure that the desired signals were not filtered out along with the unwanted signals. However, for stationary signals, both algorithms converge to the optimum Wiener solution [30]. A different technology presented by Capon in 1969 [31] was the mentioned MVDR or the Maximum Likelihood Method (MLM). This was the first attempt to automatically localize signal sources. Later in 1974, the Sample Matrix Inversion (SMI) technique, which determines the adaptive antenna array weights directly, was demonstrated in [32]. These all belong to time-dependent reference techniques, which optimize the receive antenna weights in order to identify a known sequence at the output of the antenna array. Another technique is called the spatial reference technique, based on the 
Direction Of Arrival (DOA) estimation of signal sources. Many estimation methods of wave number and DOA of signal sources can be found in [33-35]. Blind beamforming is another important beamforming technique, which does not require training sequences and any information concerning the array's geometries: for example, Constant Modulus Adaptive (CMA) beamforming in [36], Constant Modulus (CM) in [37], Spectrum self-COherent REstoral (SCORE) in [29-39] and Decision-Directed Algorithm (DDA) beamforming in [40,41]. To improve the robustness to the DOA uncertainty, the Bayesian beamformer was also proposed in $[42,43]$.

For beamforming in the downlink, the weights for each subcarrier can also be calculated iteratively. Several methods [44-46] have been presented to derive the weights for downlink based on the knowledge of those of uplink, for example, the Null Constraint (NC) weights deciding approach.

\subsection{A New NB Beamforming Technique for Downlink}

We propose a new NB method, called VDA, on top of the MVDR beamformer employed for downlink beamforming by the I-WiMAX BS.

NB technique was originally developed as a robust array beamforming technique. It was also regarded as a beampattern synthesis method. Mailoux in [47] presented a NB method by a simple modification of the covariance matrix of the received signal. While in [48], a similar NB algorithm was also presented by applying a transform to the same covariance matrix. Both methods were capable of providing a notch at the locations of interferences signals. In [49], two NB algorithms as Covariance Matrix Taper (CMT) methods are defined, and considered as effective robust adaptive beamforming techniques, imparting robustness into the adaptive pattern by a judicious choice of null placement and width. In [50,51], it was proved that NB was able to extract moving targets with less number of snapshots for the covariance matrix in sonar applications. As for wireless communication, NB was employed in a cellular communication system [52], and particularly an example was given in Space Division Multiple Access (SDMA) system for downlink beamforming [53]. In [53], three different NB schemes were compared. They were the Angular Spread (AS) based approach, high order NB and multiple nulling. All of them were shown to increase the Signal to Interference plus Noise Ratio (SINR) at the Mobile Station (MS). What is more, it also illustrated that NB for downlink beamforming was quite essential in the propagation environment with AS. Recently in [54], a constrained optimization approach for NB has been proposed, which was based on semi-definite programming. This NB method could also broaden the main beam for desired detection and consequently it achieved a better coverage. Another iterative NB method was discussed in [55] for depressing side lobes in applications of array beampattern synthesis with lower number of interference signals and high iteration speed.

We assume that the BS is equipped with a Uniform Linear Array (ULA) containing $M$ antenna elements. There are $K$ far-field SS. We first discuss VDA based on narrowband signal sources, and then we show how to calculate iteratively the whole set of weights with NB of OFDM signals.

The received signal of the $\mathrm{BS}$ is given by:

$$
\mathbf{x}(t)=\sum_{i=1}^{K} \mathbf{a}\left(\theta_{i}\right) s_{i}(t)+\mathbf{n}(t), \quad t=1,2, \ldots, L
$$

where $L$ is the number of snapshots, $\mathbf{a}\left(\theta_{i}\right)$ is the steering vector of the $i$ th signal sources in the direction of $\theta_{i}$, 


$$
\mathbf{a}\left(\theta_{i}\right)=\left(1, e^{-j \frac{2 \pi}{\lambda} d \sin \left(\theta_{i}\right)}, \ldots, e^{-j(M-1) \frac{2 \pi}{\lambda} d \sin \left(\theta_{i}\right)}\right)^{T}, \quad i=1,2, \ldots, K
$$

$T$ is the vector transpose. $s_{i}(t), i=1,2, \ldots, K$ are the signals from all SS and $\boldsymbol{n}(t)$ is the Additive White Gaussian Noise (AWGN). Parameter $d$ is the space between two array elements, and $\lambda$ is the wavelength.

The received covariance matrix at the BS equals:

$$
\boldsymbol{R}_{\text {uplink }}=\mathrm{E}\left[\boldsymbol{x} \boldsymbol{x}^{H}\right]=\sum_{i=1}^{K} \sigma_{i}^{2} \boldsymbol{a}\left(\theta_{i}\right) \boldsymbol{a}^{H}\left(\theta_{i}\right)+\sigma_{n}^{2} \boldsymbol{I}
$$

where superscript $H$ means hermitian transpose, and $\sigma_{i}^{2}, i=1,2, \ldots, K$ is the power of the $i$ th signal and $\sigma_{n}^{2}$ is the variance of the noise.

We then can estimate $\boldsymbol{a}\left(\theta_{i}\right), \sigma_{i}^{2}, \sigma_{n}^{2}$ by $\widehat{\boldsymbol{a}}\left(\theta_{i}\right), i=1,2, \ldots, K, \widehat{\sigma}_{i}^{2}, i=1,2, \ldots, K$ and $\widehat{\sigma}_{n}^{2}$, which help us to calculate the covariance matrix for the downlink beamformer of the $k$ th user,

$$
\boldsymbol{R}_{k}=\sum_{i=1, i \neq k}^{K} \widehat{\sigma}_{i}^{2} \widehat{\boldsymbol{a}}\left(\theta_{i}\right) \widehat{\boldsymbol{a}}^{H}\left(\theta_{i}\right)+\widehat{\sigma}_{n}^{2} \boldsymbol{I}
$$

The well-known CMT method is fully employed for the uplink mode. However, when it is introduced for downlink beamforming, it needs to reconstruct the covariance matrix for the $k$ th SS at downlink by estimating the DOA and powers of SS and also of the noise power, as shown in (4).

The weights of MVDR beamformer are [56]:

$$
\boldsymbol{w}_{k}=\boldsymbol{R}_{k}^{-1} \widehat{\boldsymbol{a}}\left(\theta_{k}\right) /\left(\widehat{\boldsymbol{a}}^{H}\left(\theta_{k}\right) \boldsymbol{R}_{k}^{-1} \widehat{\boldsymbol{a}}\left(\theta_{k}\right)\right)
$$

In order to produce a notch of width $W$ in each interference direction, the CMT method is required to calculate $\widetilde{\boldsymbol{R}}_{\mathrm{CMT}, k}$ by the Hadamard product of $\boldsymbol{R}_{k}$ and $\boldsymbol{T}$ :

$$
\widetilde{\boldsymbol{R}}_{\mathrm{CMT}, k}=\boldsymbol{R}_{k}^{\circ} \boldsymbol{T}
$$

where

$$
(\boldsymbol{T})_{m, n}=\operatorname{sinc}[(m-n) W / 2]
$$

represents the element of matrix $\boldsymbol{T}$, located at the $m$ th row and the $n$th column. Then by using $\widetilde{\boldsymbol{R}}_{\mathrm{CMT}, k}$ in (5) instead of $\boldsymbol{R}_{k}$, we can have the weights with NB calculated by CMT.

By using an approximation for the sinc function,

$$
\operatorname{sinc}(x) \approx 1-\frac{4}{3} \sin ^{2}\left(\frac{\pi x}{3}\right), \quad x \ll 1
$$

The null broadening $\widetilde{\boldsymbol{R}}_{k}$ in the new NB method is calculated from:

$$
\widetilde{\boldsymbol{R}}_{k}=\frac{1}{3} \boldsymbol{R}_{k}+\frac{2}{3} \boldsymbol{D}_{1} \boldsymbol{R}_{k} \boldsymbol{D}_{1}+\frac{2}{3} \boldsymbol{D}_{2} \boldsymbol{R}_{k} \boldsymbol{D}_{2}
$$

where

$$
\begin{aligned}
& \boldsymbol{D}_{1}=\operatorname{diag}\left(\left[1, \cos \left(\frac{\pi W}{3}\right), \ldots, \cos \left(\frac{\pi W(M-1)}{3}\right)\right]^{T}\right) \\
& \boldsymbol{D}_{2}=\operatorname{diag}\left(\left[1, \sin \left(\frac{\pi W}{3}\right), \ldots, \sin \left(\frac{\pi W(M-1)}{3}\right)\right]^{T}\right)
\end{aligned}
$$

$\operatorname{diag}(\boldsymbol{\beta})$ spans a diagonal matrix with each element of vector $\boldsymbol{\beta}$ on the main diagonal. 


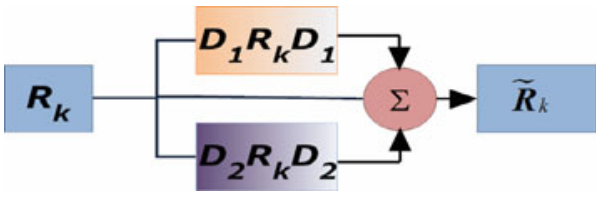

Fig. 5 Reconstructed covariance matrix for NB

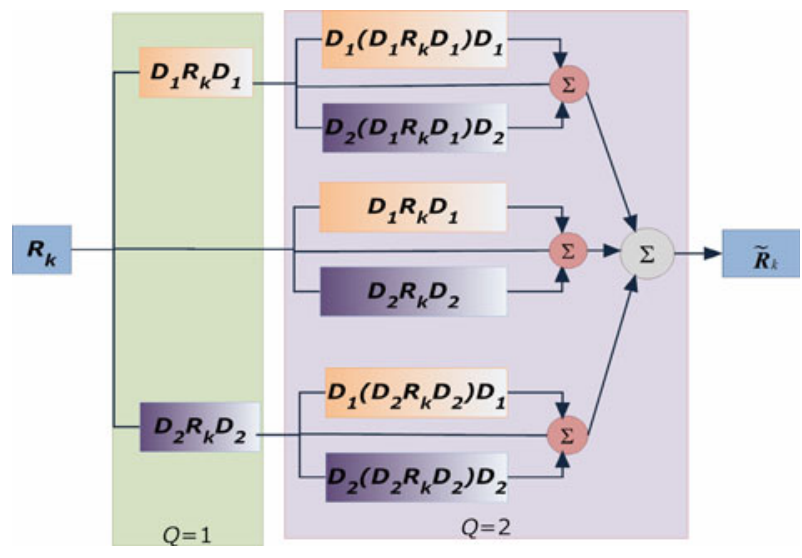

Fig. 6 The two-step iterative VDA

The proof of equation (9) is shown in Appendix A.

Similarly, if we adopt $\widetilde{\boldsymbol{R}}_{k}$ instead of $\boldsymbol{R}_{k}$ in (5), we can compute the weights $\widetilde{\boldsymbol{w}}_{k}$ of VDA. VDA can be performed by adding two directions with coherent signal sources to the received signals. Expressions (4) and (9) illustrate that the modification of the received signal at downlink of the $k$ th SS consists of 3 parts. The first is the received signal $\boldsymbol{x}(t)$ but without $\boldsymbol{a}\left(\theta_{k}\right) s_{k}(t)$, i.e., eliminating the signal from the $k$ th SS. The other two parts are considered as the coherent signals coming from directions $\cos \left(\frac{\pi W}{3}\right) e^{j \frac{2 \pi}{\lambda} \sin \left(\theta_{i}\right)}$ and $\sin \left(\frac{\pi W}{3}\right) e^{j \frac{2 \pi}{\lambda} \sin \left(\theta_{i}\right)}$ with $i \neq k, i=1,2, \ldots, K$. The reconstructed covariance matrix of $\widetilde{\boldsymbol{R}}_{k}$ is shown in Fig. 5.

In order to put deeper nulls around the required directions, the VDA can be calculated iteratively. A two-step iterative method is demonstrated in Fig. 6. To keep the same width, the starting width $W_{0}$ is chosen as

$$
W_{0}=\frac{W}{2^{Q}-1}
$$

where $Q$ is the total number of iterations.

If the weights for the downlink MVDR beamformer are calculated as $\boldsymbol{w}_{k}$ in Eq. (5), we derive

$$
\widetilde{\boldsymbol{w}}_{k}=\left(\widetilde{\boldsymbol{R}}_{k} \boldsymbol{C}^{-1}+\boldsymbol{I}\right)^{-1} \boldsymbol{w}_{k}
$$

where

$$
\boldsymbol{C}=2 \boldsymbol{D}_{1} \widetilde{\boldsymbol{R}}_{k} \boldsymbol{D}_{1}+2 \boldsymbol{D}_{2} \widetilde{\boldsymbol{R}}_{k} \boldsymbol{D}_{2}
$$

It is worth noticing that the weights of the MVDR and MMSE beamformer are both given by the Wiener solution. As a result, $\boldsymbol{w}_{k}$ of MVDR can also be calculated iteratively as $\boldsymbol{w}_{k}$ of the MMSE, which was shown in Fig. 4. For the NB case, if we let $\mathbf{U}_{k} \triangleq\left(\widetilde{\boldsymbol{R}}_{k} \boldsymbol{C}^{-1}+\boldsymbol{I}\right)^{-1}$, the different weights of different subcarriers can then be computed iteratively by: 


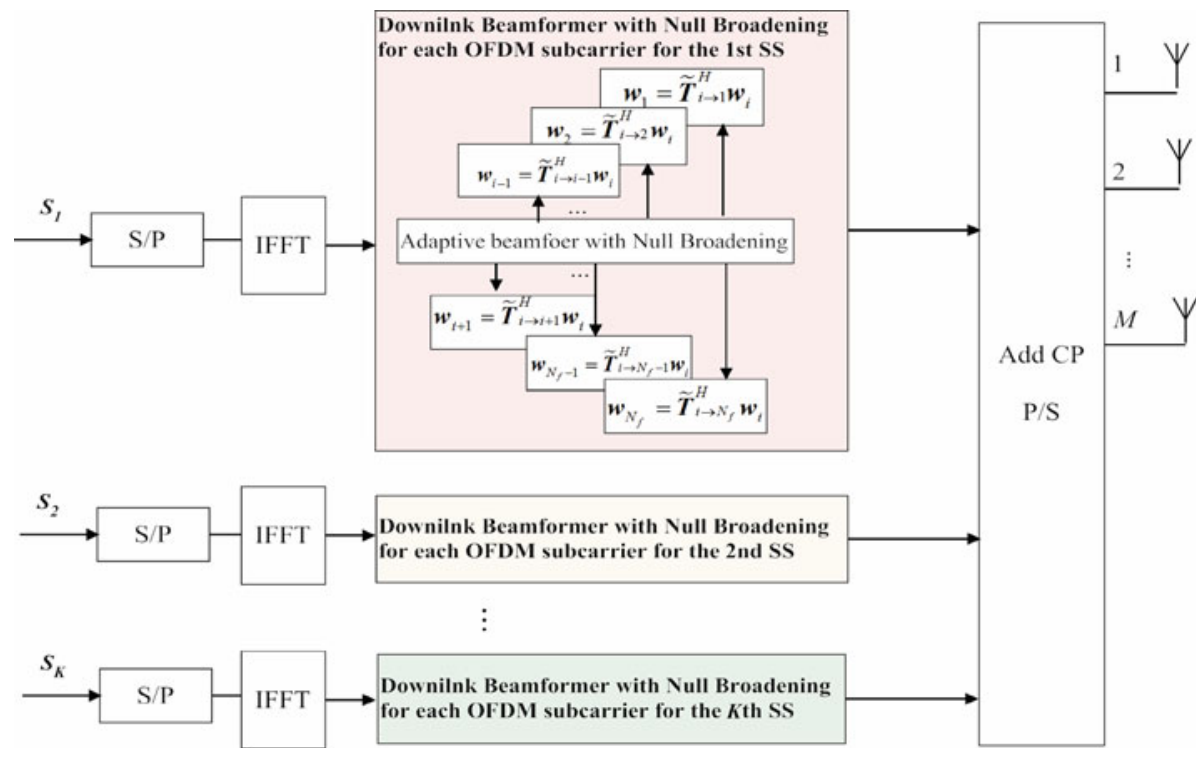

Fig. 7 Adaptive downlink OFDM beamformer with Null Broadening of I-WiMAX (S/P: Serial to Parallel; IFFT: Inverse Fast Fourier Transform; CP: Cyclic Prefix; P/S: Parallel to Serial; $N_{f}$ : number of OFDM subcarriers)

$$
\widetilde{\boldsymbol{w}}_{k, i+1}=\boldsymbol{U}_{k} \boldsymbol{T}_{i \rightarrow i+1} \boldsymbol{w}_{k, i}=\boldsymbol{U}_{k} \boldsymbol{T}_{i \rightarrow i+1} \boldsymbol{U}_{k}^{-1} \widetilde{\boldsymbol{w}}_{k, i}=\widetilde{\boldsymbol{T}}_{i \rightarrow i+1} \widetilde{\boldsymbol{w}}_{k, i}
$$

The adaptive downlink OFDM beamformer with NB is shown in Fig. 7. The weights of each subcarriers for $k$ th SS are deduced iteratively by multiplying the weights with $\widetilde{\boldsymbol{T}}_{i \rightarrow i+1}$, where $\widetilde{\boldsymbol{T}}_{i \rightarrow i+1} \triangleq \boldsymbol{U}_{k} \boldsymbol{T}_{i \rightarrow i+1} \boldsymbol{U}_{k}^{-1}$.

\subsection{Simulation Results and Radio Spectrum Efficiency Improvement}

A ULA with 16 elements at half-wavelength spacing is here taken into account $(M=16$, $d=\lambda / 2$ ). We assume that there are four SS, the four DOA's are at $\theta_{1}=-17.5^{\circ}, \theta_{2}=$ $-36.9^{\circ}, \theta_{3}=23.6^{\circ}, \theta_{4}=0\left(\sin \theta_{1}=-0.3, \sin \theta_{2}=-0.6, \sin \theta_{3}=0.4, \sin \theta_{4}=0\right)$ respectively. The SNR of the four SS are all $15 \mathrm{~dB}$. Since OFDM signals per subcarrier can be considered as narrow band, we only consider the narrow band case for simplicity. However, as can been seen in Fig. 7, the weights of VDA can be computed iteratively, meaning that the weights of different OFDM subcarriers are also easily calculated. We assume that all the SS are sharing the same spectrum band. As mentioned before, the adaptive beamformer assures for SS1 that the weights of each subcarrier should have nulls towards other three SS. The width of the notch is set to $W=0.2$. We consider $Q=2$ steps for iteratively using VDA. All the simulations are under the condition that $L=500$.

Figure 8 shows the beampattern of the two methods, i.e., CMT and VDA. Figure 8 learns that both methods can broaden nulls towards the three directions of SS2, SS3 and SS4 with the same width $W=0.2$, while directing the main beam to SS1. However, VDA can put deeper nulls to the angular around direction of SS2, SS3 and SS4 than CMT.

Figure 9 shows the CDF of the downlink power in the requested null broadening angles for SS2. We performed simulations for 5,000 times. It shows that CMT and VDA can depress 


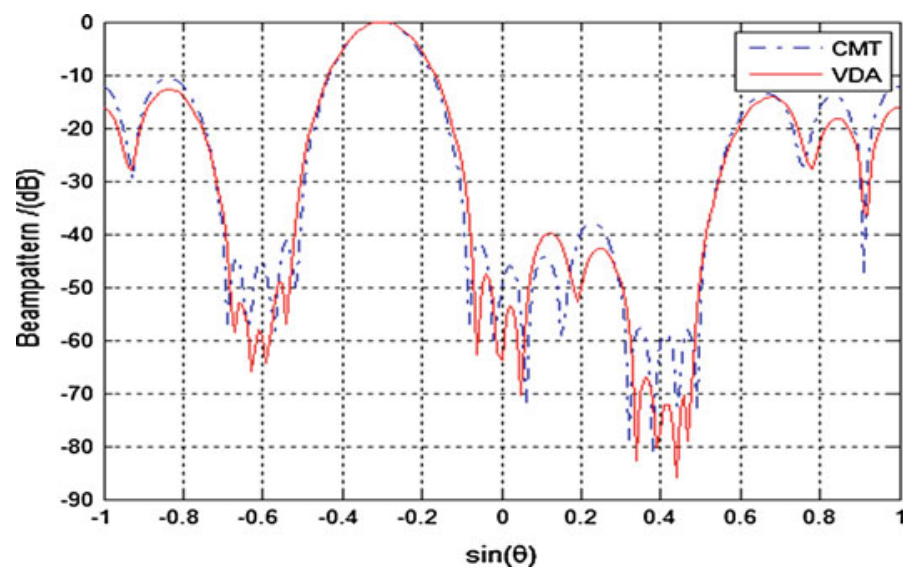

Fig. 8 Beampattern of two NB methods: CMT and VDA with for SS

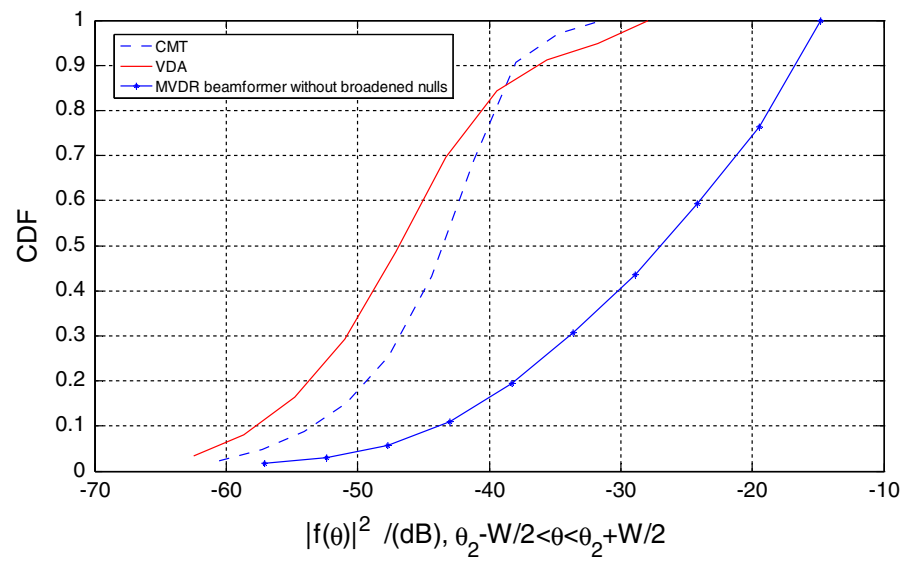

Fig. 9 CDF of the co-channel interferences depression for SS2

the power around the directions of SS2 by more than $-30 \mathrm{~dB}$. Figure 9 also illustrates that for $50 \%$ of time, VDA places the nulls with depth lower than $-47 \mathrm{~dB}$, while the CMT show deeper nulls than $-47 \mathrm{~dB}$ for only about $30 \%$. The worst performance of depressing interference at SS2 is given by adopting the MVDR beamformer without broadened nulls, which shows that there is only $8 \%$ of time that $\mathrm{SS} 2$ has a depression of co channel interference lower than $-47 \mathrm{~dB}$.

Next, we take the channel effect into the consideration. The received signal and power spectra at the SS depend on the probability density function (pdf) of the Angle of Arrival (AOA) of the scattered wave. Clarke considered a uniform AOA pdf over $[-\pi, \pi$ ) [57]. However, it has been argued and experimentally demonstrated that the scattering encountered in many environments results into a non-uniform pdf of AOA at SS [58]. Reference [59] suggests the two-parameter Von Mises pdf as a flexible and generalized model for the pdf of AOA, which includes non-istropic scattering cases, and also the isotropic one as a special case. The pdf is given as [59], 


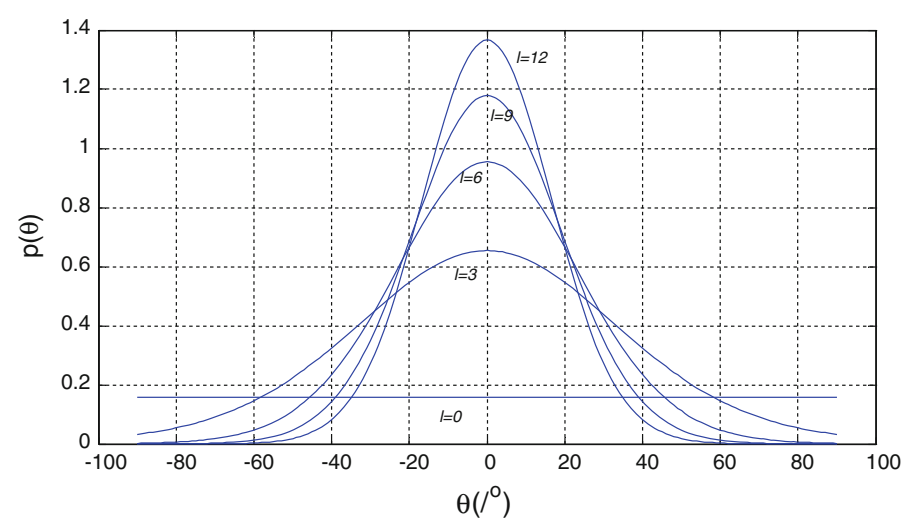

Fig. 10 Von Mises pdf for the AOA of scatter components at SS with different $l, \theta_{p}=0$

$$
p_{\Theta}(\theta)=\frac{\exp \left(l \cos \left(\theta-\theta_{p}\right)\right)}{2 \pi I_{0}(l)}, \quad \theta \in[-\pi, \pi)
$$

where $I_{0}(k)$ is the zero-order modified Bessel function, $\theta_{p}$ accounts for the main direction of the AOA scatter components. Parameter $l \geq 0$ controls the width of the AOA of scatter components. Figure 10 shows the $p_{\Theta}(\theta)$ with different $l$ for $\theta_{p}=0$.

Based on Von Mises model, point nulls which can be generated by adaptive beamformers are not sufficient enough for energy depression, because the power around the nulling direction may also leak into other SS due to spatial scattering.

Assuming that there are $K \mathrm{SS}$. The $k$ th SS receives signals of powers $\left\{E_{1}, E_{2}, \ldots, E_{K}\right\}$ from all $K$ users. The downlink Signal to Interference Ratio (SIR) for the $k$ th user is defined as [55],

$$
\eta_{k}=\frac{E_{k}}{\sum_{i=1, i \neq k}^{K} E_{i}}
$$

With $\eta_{k}$, the coverage performance can be represented by the probability distribution function $C(\gamma)=P\left\{\eta_{k}>\gamma\right\}$. In our case, $K=4$. Figures 11 and 12 compare the coverage performance of the three beamforming methods with different $l$ connected to the Von Mises pdf. It can be seen that both VDA and CMT have a higher CDF than the MVDR beamformer without NB. As a result, the coverage range is significantly enlarged by the NB methods. According to Fig. 11, the chances of VDA and CMT methods to have a SIR of SS2 lower than $-18 \mathrm{~dB}$ reaches 100 and 55\% respectively, while that of MVDR without NB is less $10 \%$ with $l=10$. When $l=7$, the CDF in total is lower than that of $l=10$; this is because the AOA pdf of the channel seen at SS is wider than that for $l=10$, as indicated in Fig. 10. However, the VDA still achieves the best among the three.

\section{Distributed Beamforming for I-WiMAX}

In this section, we introduce DB to I-WiMAX for long distance communications beyond the coverage range of one BS. As shown in Fig. 3, accessible SS are regarded as wireless relays, and consequently they form a relay network with flexible and uncertain locations of each node. We first show the feasibility of DB for I-WIMAX. Then we present a new DB method 


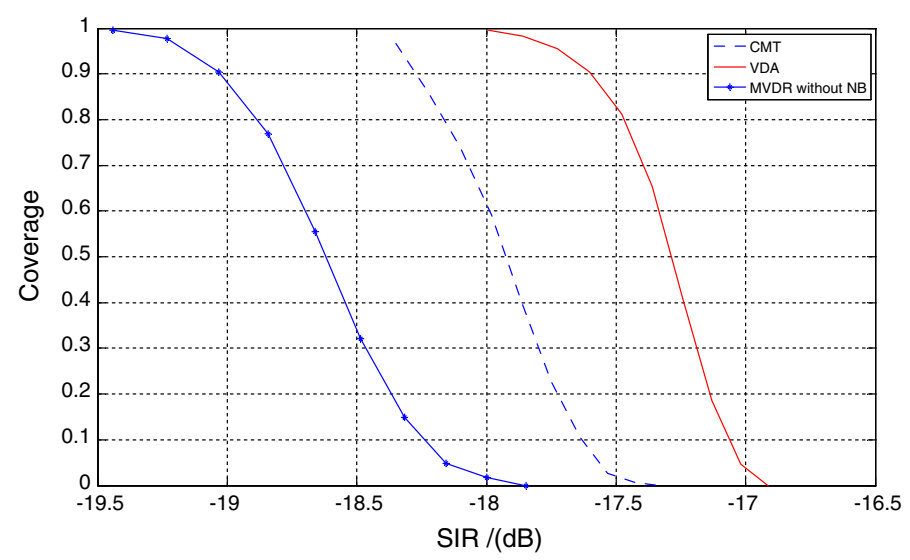

Fig. 11 Coverage performance of CMT, VDA and MVDR beamformer without NB when $l=10$

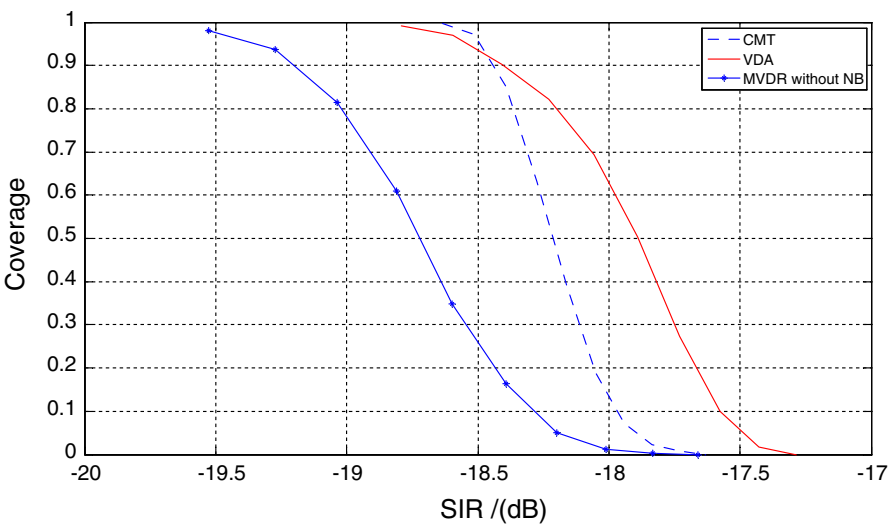

Fig. 12 Coverage performance of CMT, VDA and MVDR beamformer without NB when $l=7$

to generate different beams towards different users at the same time, rather than in different time slots. Later we show some simulation results of the method based on OFDM signals.

\subsection{DB for I-WiMAX in TDD and FDD Schemes}

WiMAX supports two duplexing schemes: Time Division Duplex (TDD) and Frequency Division Duplex (FDD). However, most WiMAX implementations will likely use TDD, because it allows the system operator to receive the most from their investment in spectrum and telecom equipment, while meeting the needs of each individual user. We demonstrate here that TDD is also more suitable for the application of DB to I-WiMAX than FDD.

Figures 13 and 14 show DB for I-WiMAX in both TDD and FDD schemes. We mark those SS, which are beyond the transmission range as Distant SS (DSS). The uplink defines the link from DSS to BS, while downlink represents the reverse. In TDD mode, as shown in Fig. 13, DSS, SS and BS require only one channel for uplink and downlink transmission in two distinct time slots. For DSS, the whole Tx and Rx procedure requires double time related to the communication session within the coverage range. In Fig. 14, it is worth noticing that for DSS and SS, the portions of the spectrum used for Tx and Rx are different; for example, 


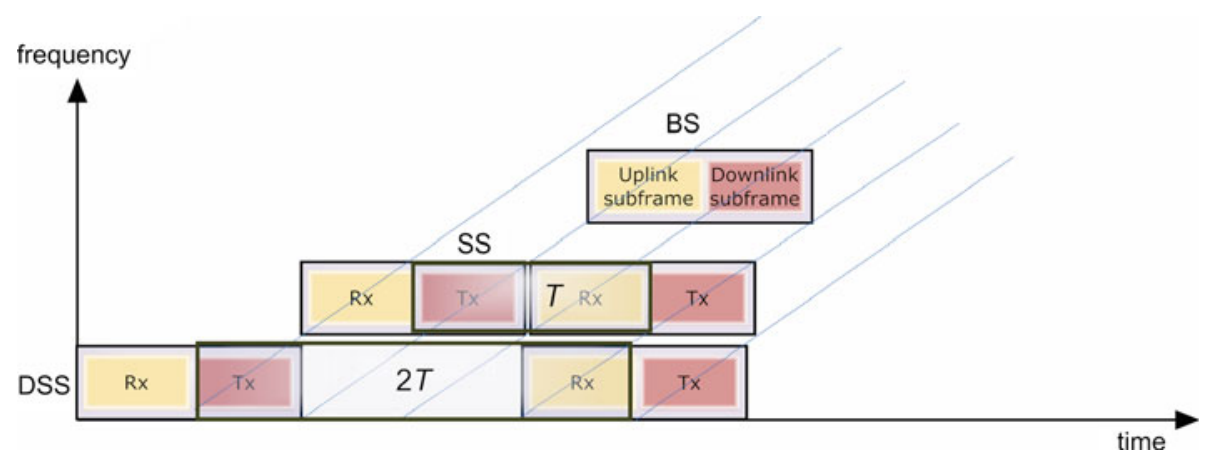

Fig. 13 DB for I-WiMAX in TDD scheme

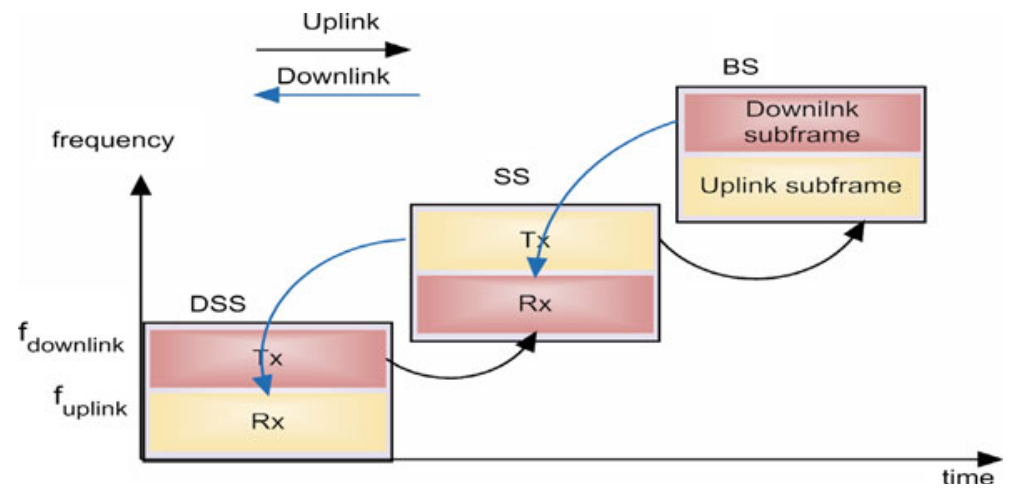

Fig. 14 DB for I-WiMAX in FDD scheme

DSS must transmit signals to SS via adopting a spectrum band of the downlink. This is a hard task for such hardware modification. Therefore, TDD scheme is more flexible and more suitable for introducing DB into I-WiMAX.

There are two most relevant relay strategies: Amplify-and-forward (AF) and decode-and forward (DF) [60]. In the AF, the relay simply transmit the scaled and phase-shifted version of their received signals, while in the DF scheme, the relay starts with decoding and then re-encode their received signals prior to retransmitting them. We can see from Fig. 13 that in the TDD mode, since Tx and Rx are performed in different time slots, both AF and DF can be adopted. In this study, we consider the AF strategy because of its low complexity.

\subsection{Multi-beam's Generating Method}

The geometry structure of the relay network in our case is illustrated in Fig. 15. The $k$ th node of the relay network is the $k$ th $\mathrm{SS}$, with the polar coordinates $\left(r_{k}, \Psi_{k}\right)$. The location coordinates of the DSS are given by $\left(A_{i}, \phi_{i}\right), i=1,2, \ldots, N_{d}$, where $N_{d}$ is the number of DSS. For a total number of SS equal to $N$, we furthermore consider the following assumptions which are the same as in [18]. The location of each SS is chosen randomly, following a uniform distribution within a disk of radius $R$. Meanwhile, we assume that all SS transmit identical energies and that the path losses of all SS are also identical, meaning no multipath fading or shadowing. Next, no mutual coupling effects in the antenna of each SS are taken 


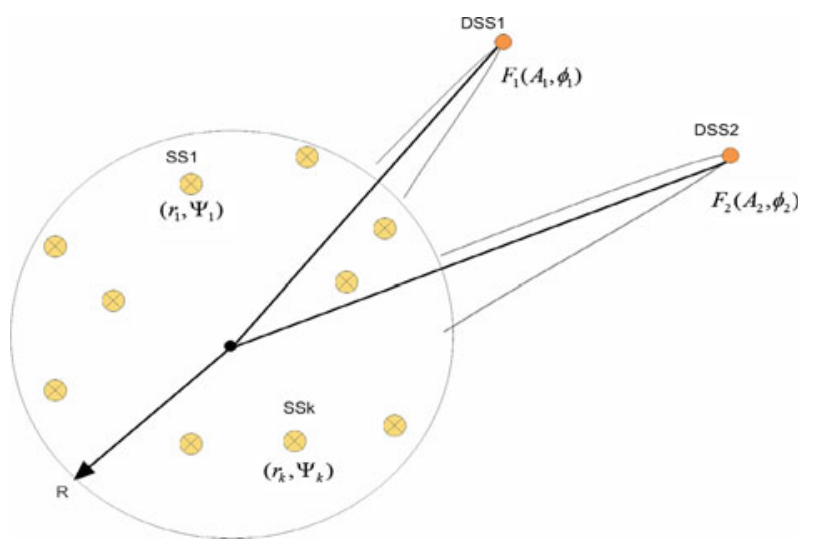

Fig. 15 Geometry structure of the relay network and DSS for DB

into account. We also assume that all the SS are perfectly synchronized so that no frequency offset or phase jitter exist.

If we chose the initial phase of each SS as

$$
\Phi_{k}=-\frac{2 \pi}{\lambda} d_{k}\left(A_{1}, \phi_{1}\right)
$$

where

$$
d_{k}\left(A_{1}, \phi_{1}\right)=\sqrt{A_{1}^{2}+r_{k}^{2}-2 A_{1} r_{k} \cos \left(\Psi_{k}-\phi_{1}\right)} \approx A_{1}-r_{k} \cos \left(\Psi_{k}-\phi_{1}\right)
$$

denotes the far-field Euclidean distance between the $k$ th SS and the 1st DSS. The far-field beampattern array factor for the set of SS is then approximated by

$$
F\left(\phi \mid r_{k}, \Psi_{k}\right) \approx \frac{1}{N} \sum_{k=1}^{N} \exp \left\{j \frac{2 \pi}{\lambda} r_{k}\left[\cos \left(\phi_{1}-\Psi_{k}\right)-\cos \left(\phi-\Psi_{k}\right)\right]\right\}
$$

The locations of SS follow the uniform distribution over the disk of radius $R$. Thus, the probability density functions (pdf) are

$$
f_{r_{k}}(r)=\frac{2 r}{R^{2}}, \quad 0 \leq r<R ; f_{\Psi_{k}}(\Psi)=\frac{1}{2 \pi}, \quad-\pi \leq \Psi_{k}<\pi
$$

By introducing $\widetilde{r_{k}} \triangleq r_{k} / R, \widetilde{\Psi}_{k} \triangleq \Psi_{k}-\left(\phi_{1}+\phi\right) / 2, z_{k} \triangleq \widetilde{r_{k}} \sin \left(\widetilde{\Psi}_{k}\right)$, the compound random variable $z_{k}$ has the following pdf, as shown in [18],

$$
f_{z_{k}}(z)=\frac{2}{\pi} \sqrt{1-z^{2}}, \quad-1 \leq z<1
$$

Then the array factor in Eq. (20) can be written as

$$
F\left(\phi \mid z_{k}\right)=\frac{1}{N} \sum_{k=1}^{N} \exp \left[-j 4 \pi \widetilde{R} \sin \left(\frac{\phi-\phi_{1}}{2}\right) z_{k}\right]
$$


where $\widetilde{R} \triangleq R / \lambda$ is the radius of the disk normalized by the wavelength. The far field beampattern can be defined as

$$
P\left(\phi \mid z_{k}\right) \triangleq\left|F\left(\phi \mid z_{k}\right)\right|^{2}=\frac{1}{N}+\frac{1}{N^{2}} \sum_{k=1}^{N} \exp \left(-j \alpha(\phi) z_{k}\right) \sum_{l=1, l \neq k}^{N} \exp \left(j \alpha(\phi) z_{l}\right)
$$

where $\alpha(\phi)=4 \pi \widetilde{R} \sin \left(\frac{\phi-\phi_{1}}{2}\right)$.

The average array beampattern of the random distributed SS becomes [18]

$$
P_{a v}(\phi) \triangleq \mathrm{E}_{z}\left[P\left(\phi \mid z_{k}\right)\right]=\frac{1}{N}+\left(1-\frac{1}{N}\right) \mu^{2}(\phi)
$$

where

$$
\mu(\phi)=\left|\frac{2 J_{1}(\alpha(\phi))}{\alpha(\phi)}\right|
$$

and $J_{n}(\cdot)$ stands for the $n$th order Bessel function of the first kind.

If we adopt the initial phase as given in Eq. (18), it means that the average array pattern generated by the SS relay network towards $\left(A_{1}, \phi_{1}\right)$ can be expressed by Eq. (25). However, we can set an initial phase for SS different from Eq. (18), as for example has been shown in [18]. For adopting initial phases as given by Eq. (18), the synchronization of each SS requires an accurate knowledge on the distance, relative to the wavelength, between each node and the absolute distance. It is then possible to realize a closed-loop case as in self phasing arrays, and may be achieved by the use of reference signals such as comes from the Global Positioning System (GPS) [18].

The average beampattern in Eq. (25) does not show the probability for a certain amount of energy that may be received at different $\phi$. This problem has still not yet been addressed. We therefore give now the CDF of $P\left(\phi \mid z_{k}\right), \operatorname{Prob}\left\{P\left(\phi \mid z_{k}\right) \mid P\left(\phi \mid z_{k}\right) \leq P_{0}\right\}$ when $N$ is large. In Eq. (23), when $N$ is large we know that according to the Central Limit Theorem, $F\left(\phi \mid z_{k}\right)$ satisfies the Gaussian distribution, i.e. $F\left(\phi \mid z_{k}\right) \sim \mathrm{N}\left(\mu(\phi), \frac{1-\mu^{2}(\phi)}{N}\right)$. Then the absolute value of $F\left(\phi \mid z_{k}\right)-\mu(\phi)$ obeys the Rayleigh distribution. Finally, we obtain

$$
\begin{aligned}
& \operatorname{Prob}\left\{P\left(\phi \mid z_{k}\right) \mid P\left(\phi \mid z_{k}\right)>P_{0}\right\}=1-\operatorname{Prob}\left\{P\left(\phi \mid z_{k}\right) \mid P\left(\phi \mid z_{k}\right) \leq P_{0}\right\} \\
& \quad=1-\left[F\left(|\mu(\phi)|+\sqrt{P_{0}}\right)-\frac{\operatorname{sgn}\left(|\mu(\phi)|-\sqrt{P_{0}}\right)+1}{2} F\left(|\mu(\phi)|-\sqrt{P_{0}}\right)\right]
\end{aligned}
$$

where $F\left(x_{0}\right)=\operatorname{Prob}\left(x \mid x \leq x_{0}\right)=1-\exp \left(-x_{0}^{2} N /\left(1-\mu^{2}(\phi)\right)\right)$ for $x \geq 0$, is the CDF of the Rayleigh distribution of $P\left(\phi \mid z_{k}\right)$, and $\operatorname{sgn}(x)=|x| / x$.

The proof of equation (27) is shown in Appendix B.

Figure 16 shows the result of Eq. (27), indicating the probability distribution of the energy larger than $P_{0}$ with spreading over different $\phi$. We here considered different $\widetilde{R}$ with $N=256$, and $P_{0}=0.8$. We assumed only one DSS and a DOA of $\phi=0$. From Fig. 16, we can see that with larger $\widetilde{R}$, at the same probability of more than $80 \%$ the energy focuses closer to the direction of DSS1.

Using Eqs. (18) and (25), it is easy to generate one beam towards one direction at the time: consequently the forming of more beams needs more time. Next, we present two methods to form more than one beam in space at the same time. Those beams belong to different DSS users when they receive signals within the same spectrum band. 


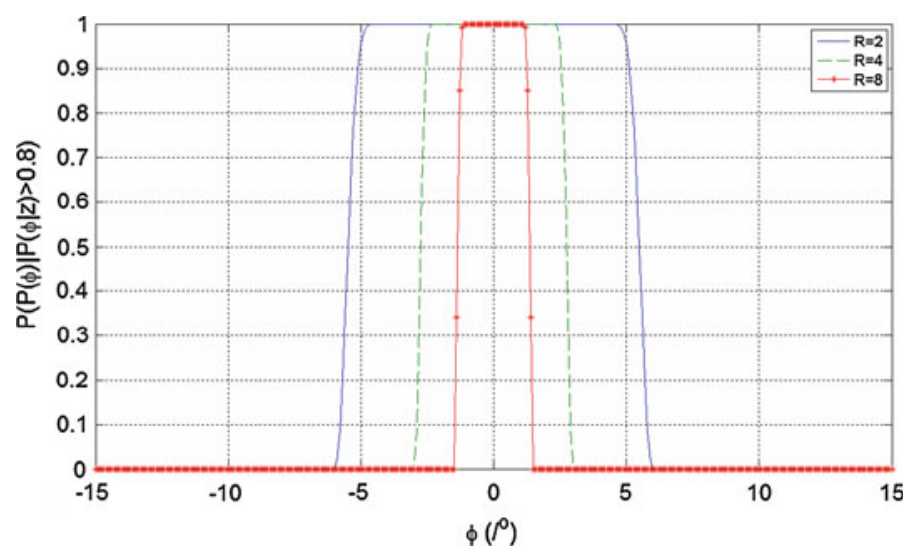

Fig. 16 The probability of $P\left(\phi \mid z_{k}\right)>0.8$

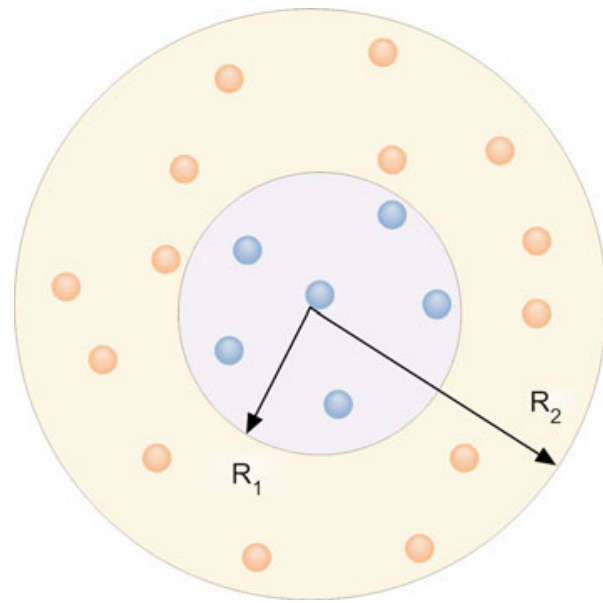

Fig. 17 The proposed Multi-beam generating method of DB by separation of SS into a ring range and a circle range

\subsubsection{Beampattern of Nodes Distributed in a Ring Range}

We here discuss the case when nodes of networks are distributed in a ring range, rather than in a circle. Figure 17 shows the structure and parameters.

We assume that the locations of SS which are in the ring range follow the uniform distribution over the ring in the radius interval $R_{2}-R_{1}$. Thus, the pdf's are

$$
f_{r_{k}}(r)=\frac{2 r}{R_{2}^{2}-R_{1}^{2}}, \quad R_{1} \leq r_{k}<R_{2} ; \quad f_{\Psi_{k}}(\Psi)=\frac{1}{2 \pi}, \quad-\pi \leq \Psi_{k}<\pi
$$

Similarly, we can write for the average far-field beampattern of SS distributed in a ring range

$$
P_{a v}^{\text {ring }}(\phi)=\frac{1}{N}+\left(1-\frac{1}{N}\right) \frac{\left[\mu(\phi)-\xi^{2} v(\phi)\right]^{2}}{\left(1-\xi^{2}\right)^{2}}
$$

where $\xi=\frac{R_{1}}{R_{2}}, v(\phi)=\frac{2 J_{1}(\xi \alpha(\phi))}{\xi \alpha(\phi)}$. 
The proof of Eq. (29) is shown in Appendix C.

Then by separating SS into different groups, we synchronize them with different initial phases. For example, all SS located in the inner circle will have the initial phases of $\Phi_{k}^{\text {circle }}=-\frac{2 \pi}{\lambda} d_{k}\left(A_{1}, \phi_{1}\right)$, while for those SS, which are in the ring range, we adopt different initial phases of $\Phi_{k}^{r i n g}=-\frac{2 \pi}{\lambda} d_{k}\left(A_{2}, \phi_{2}\right)$. Then we have two beams towards both $\left(A_{1}, \phi_{1}\right)$ and $\left(A_{2}, \phi_{2}\right)$. If we need to direct beams towards more than two directions, we can separate SS, located in the outer circle of Fig. 17, into several different ring ranges with different radii.

\subsubsection{DB with Nodes Having Randomly Selecting Phases}

Another method to display more than one beam at the same time slot is to let the SS randomly chose which DSS to serve. If the $k$ th SS chose the $i$ th DSS to transmit signals, it will set the initial phases as $\Phi_{k}=-\frac{2 \pi}{\lambda} d_{k}\left(A_{i}, \phi_{i}\right), i=1,2, \ldots, N_{d}$. The chance that SS chose DSS is equal to $1 / N_{d}$. In this case, the far-field beampattern of the SS relay network yields

$$
F\left(\phi \mid \eta_{k}, r_{k}, \Psi_{k}\right)=\frac{1}{N} \sum_{k=1}^{N} \exp \left[-j 4 \pi \widetilde{R} \sin \left(\frac{\phi-\eta_{k}}{2}\right) \sin \left(\frac{2 \Psi_{k}-\phi-\eta_{k}}{2}\right)\right]
$$

where $\eta$ is a discrete random variable, which has the following pdf

$$
\operatorname{Prob}\left(\eta \mid \eta=\phi_{i}\right)=\frac{1}{N_{d}}, \quad i=1,2, \ldots, N_{d}
$$

Then the average array beampattern of the random distributed SS with random selected initial phased becomes

$$
\begin{aligned}
& P_{a v}(\phi) \triangleq \mathrm{E}_{z}\left[P\left(\phi \mid \eta_{k}, r_{k}, \Psi_{k}\right)\right] \\
& =\sum_{i=1}^{N_{d}} \mathrm{E}_{z}\left[P\left(\phi \mid r_{k}, \Psi_{k}, \eta_{k}=\phi_{i}\right)\right] \cdot \operatorname{Prob}\left(\eta \mid \eta=\phi_{i}\right) \\
& =\frac{1}{N}+\frac{1}{N_{d}} \sum_{i=1}^{N_{d}}\left[\left(1-\frac{1}{N}\right) \mu_{i}^{2}(\phi)\right]=\frac{1}{N}+\left(1-\frac{1}{N}\right)\left[\frac{1}{N_{d}} \sum_{i=1}^{N_{d}} \mu_{i}(\phi)\right]^{2}
\end{aligned}
$$

where

$$
\mu_{i}(\phi)=\frac{2 J_{1}\left(\alpha_{i}(\phi)\right)}{\alpha_{i}(\phi)}, \quad \alpha_{i}(\phi)=4 \pi \widetilde{R} \sin \left(\frac{\phi-\phi_{i}}{2}\right), \quad i=1,2, \ldots, N_{d} .
$$

The results of the two multi-beam generating methods discussed above are shown in Fig. 18. We here consider the case $N=256, \widetilde{R}_{2}=2$. For the first multi-beam generating method, $\xi=\frac{R_{1}}{R_{2}}=\frac{1}{3}$. We chose the SS in the inner circle to form a beam towards $\phi_{1}=0$, while the rest direct the beam at direction of $\phi_{2}=60^{\circ}$. We can see that with both methods, two beams towards two required directions operate successfully. The beampattern of SS, distributed in a ring range, has higher sidelobes than that of a circle range. In the ring range case, the number of the SS is about $N \xi^{2}$, while that of the rest SS in a circle is $N\left(1-\xi^{2}\right)$. According to [18], the sidelobe approaches $1 / N$ as the beam angle moves away from the target direction, which explains the $8 \mathrm{~dB}$ difference in sidelobe asymptote of the two beam patterns formed by SS in a circle and in a ring range. When the SS randomly chooses its initial phases, the beampattern tends to have two beams towards $\phi_{1}, \phi_{2}$ with half of the energy each. This is the result of an equally chance of selecting different initial phases. 


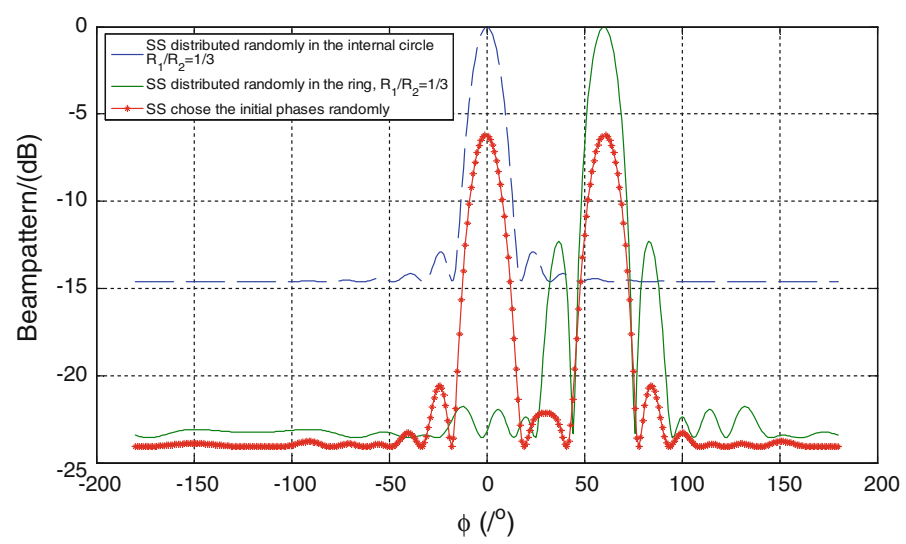

Fig. 18 DB with beampatterns of two multi-beam generating methods $\phi_{1}=0, \phi_{2}=60^{\circ}$

Applying the DB method to the OFDM signal, different subcarriers are required to adopt different initial phases, because they operate at different frequency bands. The initial phases based on Eq. (18) for the ith subcarrier of the $k$ th mode can be expressed as:

$$
\Phi_{k, i}=-\frac{2 \pi}{\lambda_{i}} d_{k}\left(A_{1}, \phi_{1}\right)=-\frac{2 \pi}{c}\left(f_{0}+(i-1) \Delta f\right) d_{k}\left(A_{1}, \phi_{1}\right), \quad i=1,2, \ldots, N_{f}
$$

We assumed here $N_{f}$ OFDM subcarriers, with frequency spacing $\Delta f$ between two adjacent subcarriers, and $f_{0}$ is the starting frequency of the operating band.

From Eq. (25), the average beampattern of the $i$ th subcarrier can be written as

$$
P_{a v, i}(\phi) \triangleq \mathrm{E}_{z}\left[P_{i}\left(\phi \mid z_{k}\right)\right]=\frac{1}{N}+\left(1-\frac{1}{N}\right)\left[\mu^{(i)}(\phi)\right]^{2}
$$

where

$$
\begin{aligned}
\mu^{(i)}(\phi) & =\left|\frac{2 J_{1}\left(\alpha^{(i)}(\phi)\right)}{\alpha^{(i)}(\phi)}\right|, \\
\alpha^{(i)}(\phi) & =4 \pi \widetilde{R_{0}}\left(1+\frac{\Delta f(i-1)}{f_{0}}\right) \sin \left(\frac{\phi-\phi_{i}}{2}\right), \quad i=1,2, \ldots, N_{d}, \\
\widetilde{R_{0}} & =\frac{R}{c} f_{0}
\end{aligned}
$$

For WiMAX, there are three frequency bands defined in the standard, 2.5 and $3.5 \mathrm{GHz}$ licensed spectrum, and the unlicensed $5 \mathrm{GHz}$ spectrum [61]. The subcarrier spacing of WiMAX is always $10.94 \mathrm{kHz}$, which means when the available bandwidth increases, the number of subcarriers will also increases. WiMAX supports the channel bandwidth of $1.25,5,10$, and $20 \mathrm{MHz}$. We assume that $f_{0}=3.5 \mathrm{GHz}$, and the channel band is $1.25 \mathrm{MHz}$. Therefore, 128 subcarriers will be used. Then

$$
\frac{\Delta f(i-1)}{f_{0}} \leq \frac{10.94 \times 127}{3.5} \times 10^{-6} \approx 4 \times 10^{-4} \ll 1, \quad i=1,2, \ldots, N_{d},
$$

Therefore,

$$
\widetilde{R_{0}}\left(1+\frac{\Delta f(i-1)}{f_{0}}\right) \approx \widetilde{R_{0}}
$$


It means that in this case the average beampatterns of the all subcarriers are approximately the same.

Meanwhile, since the coverage of mobile WiMAX in an out door scenario is about $1 \mathrm{~km}$, we can choose the radius $R$ of the circle, where the SS are distributed, as large as $1 \mathrm{~km}$ to include more nodes in the relay network. Meanwhile we estimate $\widetilde{R_{0}}=\frac{R}{c} f_{0} \gg 1$. It has been discussed in [18] that the 3 - $\mathrm{dB}$ bandwidth of the average beampattern equals

$$
\phi_{a v}^{3 d B}=2 \arcsin \left(\frac{0.1268}{\widetilde{R_{0}}}\right)
$$

As a result, in our case of WiMAX, the 3-dB bandwidth of the average main beam will be very small, in other words, the main beam is quite narrow. It is desirable to achieve a narrow main beam. However, when DSS is moving or the location of DSS is not accurate enough, too narrow beams may cause problems, as the DSS may not be covered by the main beam.

\section{Conclusions}

I-WiMAX, as a new maritime wireless communication system, promises a large coverage range, high data rates, efficient spectrum usage, and reliable communications in sea/lake scenarios. It consists of SR and mobile WiMAX. SR introduces two beamforming techniques, $\mathrm{AB}$ and $\mathrm{DB}$, for ultimate efficient spectrum utilization and large coverage.

AB for uplink can spatially select the receiving signals of interested SS and alleviate cochannel interferences by directing the main beam towards the interested SS and nulls towards others. For downlink adaptive beamforming, the NB method is introduced in I-WiMAX to display spread nulls in a certain range of directions. We propose a new NB method, called VDA which is based on the MVDR beamformer. The VDA guarantees deeper nulls than CMT, while keeping the same width, and consequently decreasing co-channel interferences effectively and improving the coverage range significantly. What is more, the weights of different OFDM subcarriers with NB can be calculated iteratively, which reduces the computational complexity. When taking into account a spatial channel model, as is seen at the $\mathrm{SS}$, it proves that NB is an essential part of I-WiMAX when serving reliable links to more than one SS within the same spectrum bands.

For SS (far) beyond the possible communication coverage range, we propose a relay network formed by those accessible SS to transmit the signals further to distant SS, rather than involving another BS. Due to the uncertainty of the location of the relay nodes, DB is employed for the SS relay network to transmit signals collaboratively after adopting different initial phases. We showed that the TDD mode of WiMAX is more suitable than FDD. However, sharing the spectrum requires directing more than one beam at the same time towards different DSS. Therefore, we proposed two methods of generating multiple beams. Both of them are able to focus transmitting energy on the requested directions. As a result, different DSS can receive signals from BS constructively with efficient spectrum utilization.

\section{Appendix A}

Proof of Equation (9)

From Eqs. (6) and (7), the element of the mth row and nth column of the covariance matrix can be written as 


$$
\left(\widetilde{\boldsymbol{R}}_{\mathrm{CMT}, k}\right)_{m, n}=\left(\boldsymbol{R}_{k}\right)_{m, n} \operatorname{sinc}\left[\frac{(m-n) W}{2}\right]
$$

Then by using the approximation proposed in Eq. (8), we can write

$$
\begin{aligned}
& \left(\widetilde{\boldsymbol{R}}_{\mathrm{CMT}, k}\right)_{m, n} \approx\left(R_{k}\right)_{m, n}\left[1-\frac{4}{3} \sin ^{2}\left(\frac{\pi(m-n) W}{2 \times 3}\right)\right] \\
& =\frac{1}{3}\left(\boldsymbol{R}_{k}\right)_{m, n}+\frac{2}{3} \sum_{i=0}^{N-1} \widehat{\sigma}_{i}^{2} e^{j \frac{2 \pi}{\lambda} x_{m} \sin \theta_{i}} \cos \left(\frac{\pi W}{3}\right) e^{-j \frac{2 \pi}{\lambda} x_{n} \sin \theta_{i}} \cos \left(\frac{\pi W}{3}\right) \\
& \quad+\frac{2}{3} \sum_{i=0}^{N-1} \widehat{\sigma}_{i}^{2} e^{j \frac{2 \pi}{\lambda} x_{m} \sin \theta_{i}} \sin \left(\frac{\pi W}{3}\right) e^{-j \frac{2 \pi}{\lambda} x_{n} \sin \theta_{i}} \sin \left(\frac{\pi W}{3}\right) \\
& \triangleq \widetilde{\boldsymbol{R}}_{k}
\end{aligned}
$$

where $x_{m}, x_{n}$ are the element locations of array antenna.

If we adopt the definitions of $\boldsymbol{D}_{1}, \boldsymbol{D}_{2}$ in Eqs. (10) and (11), $\widetilde{\boldsymbol{R}}_{k}$ can be easily written as $\widetilde{\boldsymbol{R}}_{k}=\frac{1}{3} \boldsymbol{R}_{k}+\frac{2}{3} \boldsymbol{D}_{1} \boldsymbol{R}_{k} \boldsymbol{D}_{1}+\frac{2}{3} \boldsymbol{D}_{2} \boldsymbol{R}_{k} \boldsymbol{D}_{2}$, which is shown in Eq. (9).

\section{Appendix B}

Proof of Equation (27)

If we let

$$
\begin{aligned}
& X \triangleq F\left(\phi \mid z_{k}\right) \\
& Y \triangleq\left|F\left(\phi \mid z_{k}\right)-\mu(\phi)\right|
\end{aligned}
$$

we then obtain

$$
\begin{aligned}
& X \sim \mathrm{N}\left(\mu(\phi), \quad \frac{1-\mu^{2}(\phi)}{N}\right) \\
& Y \sim \operatorname{Rayleigh}\left(\sigma_{r}^{2}\right)
\end{aligned}
$$

According to the properties of the Rayleigh distribution,

$$
E\left(y^{2}\right)=\sigma_{r}^{2} 2^{2 / 2} \Gamma\left(1+\frac{1}{2}\right)=2 \sigma_{r}^{2}
$$

On the other hand,

$$
E\left(y^{2}\right)=\mathrm{E}\left[(x-\mu(\phi))^{2}\right]=\frac{1-\mu^{2}(\phi)}{N}
$$

Then from Eqs. (B-1) and (B-2), we obtain

$$
\sigma_{r}^{2}=\frac{1-\mu^{2}(\phi)}{2 N}
$$

We calculate the probability $\operatorname{Prob}\left\{P\left(\phi \mid z_{k}\right) \mid P\left(\phi \mid z_{k}\right)>P_{0}\right\}$, which is equal to

$$
1-\operatorname{Prob}\left\{P\left(\phi \mid z_{k}\right) \mid P\left(\phi \mid z_{k}\right) \leq P_{0}\right\} .
$$

We next discuss the outcome for the different cases. 
If $|\mu(\phi)|>\sqrt{P_{0}}, \quad y \in\left[|\mu(\phi)|-\sqrt{P_{0}}, \quad|\mu(\phi)|+\sqrt{P_{0}}\right]$,

then

$$
\operatorname{Prob}\left(x \mid x^{2} \leq P_{0}\right)=F\left(|\mu(\phi)|+\sqrt{P_{0}}\right)-F\left(|\mu(\phi)|-\sqrt{P_{0}}\right)
$$

If $|\mu(\phi)|<\sqrt{P_{0}}, y \in\left[0,|\mu(\phi)|+\sqrt{P_{0}}\right]$,

then

$$
\operatorname{Prob}\left(x \mid x^{2} \leq P_{0}\right)=F\left(|\mu(\phi)|+\sqrt{P_{0}}\right)
$$

where

$$
\begin{aligned}
F\left(y_{0}\right)=\operatorname{Prob}\left(y \mid y \leq y_{0}\right) & =1-\exp \left(-y_{0}^{2} / 2 / \sigma_{r}^{2}\right) \\
& =1-\exp \left(-y_{0}^{2} N /\left(1-\mu^{2}(\phi)\right)\right)
\end{aligned}
$$

To combine (B-4) and (B-5), it is easy to verify Eq. (27)

$$
\begin{aligned}
\operatorname{Prob}\left\{x^{2} \mid x^{2}>P_{0}\right\}= & 1-\operatorname{Prob}\left\{x^{2} \mid x^{2} \leq P_{0}\right\} \\
= & 1-\left[F\left(|\mu(\phi)|+\sqrt{P_{0}}\right)-\frac{\operatorname{sgn}\left(|\mu(\phi)|-\sqrt{P_{0}}\right)+1}{2}\right. \\
& \left.\times F\left(|\mu(\phi)|-\sqrt{P_{0}}\right)\right]
\end{aligned}
$$

\section{Appendix C}

Proof of Equation (29)

We define

$$
u_{k} \triangleq \frac{r_{k}}{R_{2}} \sin \frac{\phi+\phi_{0}-2 \Psi_{k}}{2}
$$

By adopting the method in [18], we can calculate the pdf of random variable $u_{k}$,

$$
f_{U_{k}}\left(u_{k}\right)= \begin{cases}\frac{1}{\pi\left(1-\xi^{2}\right)} \sqrt{1-u_{k}^{2}}, & \xi<u_{k} \leq 1 \\ \frac{1}{\pi\left(1-\xi^{2}\right)}\left(\sqrt{1-u_{k}^{2}}-\sqrt{\xi^{2}-u_{k}^{2}}\right), & \left|u_{k}\right| \leq|\xi| \\ \frac{1}{\pi\left(1-\xi^{2}\right)} \sqrt{1-u_{k}^{2}}, & -1 \leq u_{k}<-\xi\end{cases}
$$

Then we can obtain the expectation of $\exp \left(-j \alpha(\phi) u_{k}\right)$ by

$$
\begin{aligned}
& \mathrm{E}\left[\exp \left(-j \alpha(\phi) u_{k}\right)\right] \\
& =\frac{1}{\pi\left(1-\xi^{2}\right)}\left[\int_{-1}^{1} \sqrt{1-u_{k}^{2}} \exp \left(-j \alpha(\phi) u_{k}\right) d u_{k}-\int_{-\xi}^{\xi} \sqrt{\xi^{2}-u_{k}^{2}} \exp \left(-j \alpha(\phi) u_{k}\right) d u_{k}\right] \\
& =\frac{1}{1-\xi^{2}}\left[\mu(\phi)-\xi^{2} \frac{2 J_{1}(\alpha(\phi) \xi)}{(\alpha(\phi) \xi)}\right]=\frac{1}{1-\xi^{2}}\left[\mu(\phi)-\xi^{2} v(\phi)\right]
\end{aligned}
$$


From Eqs. (24) and (25), we derive

$$
\begin{aligned}
P_{a v}^{r i n g}(\phi) & =\mathrm{E}\left[P\left(\phi \mid z_{k}\right)\right] \\
& =\frac{1}{N}+\frac{1}{N^{2}} \mathrm{E}\left[\sum_{k=1}^{N} \exp \left(-j \alpha(\phi) u_{k}\right) \sum_{l=1, l \neq k}^{N} \exp \left(j \alpha(\phi) u_{l}\right)\right] \\
& =\frac{1}{N}+\frac{N-1}{N}\left[\mathrm{E}\left(\exp \left(-j \alpha(\phi) u_{k}\right)\right)\right]^{2} \\
& =\frac{1}{N}+\frac{N-1}{N} \frac{\left[\mu(\phi)-\xi^{2} v(\phi)\right]^{2}}{\left(1-\xi^{2}\right)^{2}}
\end{aligned}
$$

which shows the same results as in Eq. (29).

Open Access This article is distributed under the terms of the Creative Commons Attribution Noncommercial License which permits any noncommercial use, distribution, and reproduction in any medium, provided the original author(s) and source are credited.

\section{References}

1. Lian, X., Nikookar, H., \& Ligthart, L. (2010). Application of Green Radio to Maritime Coastal/Lake Communications and Locationing Introducing Intelligent WiMAX (I-WiMAX). In Towards green ICT (pp. 287-305). Aalborg: River Publisher.

2. Claudio, E. D., \& Parisi, R. (2001). WAVES: Weighted average of signal subspaces for robust wideband direction finding. IEEE Transactions on Signal Processing, 49(10), 2179-2190.

3. Barrett, M., \& Arnott, R. (1994). Adaptive antennas for mobile communications. IEE Electronic \& Communications Engineering Journal, pp. 203-214.

4. Mizuno, M., \& Takeo, O. (2007). Application of adaptive array antennas to radio communications. Electronics and Communications in Japan, 77(2), 48-59.

5. Tsoulos, G. V., Beach, M. A., \& Swales, S. C. (1997). On the sensitivity of the capacity enhancement of a TDMA system with adaptive multibeam antennas. In Proceedings of IEEE 47th VTC (Vol. 1, pp. 165-169). Phoenix, USA, 4-7 May, 1997.

6. Nicoli, M., Sala, M., Simeone, O., Sampietro, L., \& Santacesaria, C. (2007). Adaptive array processing for time-varying interference mitigation in IEEE802.16 systems. In Proceedings of PIMRC (pp. 1-5). Athens, Greece, 3-7 September, 2007.

7. Porter, J. W., Kepler, J. F., Krauss, T. P., Vook, F. W., Blankenship, T. K., Desai, V., et al. (2007). An experimental adaptive beamforming system for the IEEE802.16e-2005 OFDMA downlink. In Proceedings of IEEE RWS (pp. 475-478). Orlando, USA, 22-25 January, 2007.

8. Desai, V., Kepler, J. F., \& Vook, W. (2007). Field data showing the downlink adaptive beamforming gains in an experimental IEEE802.16e-2005 OFDMA system. In Proceedings of IEEE RWS (pp. 619-622). Orlando, USA, 22-25 January, 2007.

9. Li, Y., \& Kenyon, D. (2005). An examination of the processing complexity of an adaptive antenna system for WiMAX. In Proceedings of the 2nd IEE/EURASIP conference on DSPenabledRadio (pp. 7-13). Glasgow, UK, 19-20 September, 2005.

10. Ogawa, Y., \& Ohgane, T. (1996). Adaptive antennas for future mobile radio. IEICE Transaction Fundamentals, E79-A(7), 961-967.

11. Swales, S. C., Beach, M. A., Edwards, D. J., \& McGeehan, J. P. (1990). The performance enhancement of multibeam adaptive base-station antennas for cellular land mobile radio systems. IEEE Transaction on Vehicle Technology, 39(1), 56-67.

12. Aderson. N., \& P. Howard. (1997). Technology and transceiver architecture considerations for adaptive antenna systems. In Proceedings of ACTS Summit (pp. 965-970), 1997.

13. Strandell, J., Wennstrom, M., Rydberg, A., Oberg, T., Gladh, O., Rexberg, L., et al. (1997). Experimental Evaluation of an adaptive antenna for TDMA mobile telephony system. In Proceedings of PIMRC (pp. 79-84). Helsinki, Finland, 1-4 September, 1997.

14. Monot, J. J., Thibault, J., Chevalier, P., Pipon, F., Mayrargue S., \& Levy, A. (1997). A fully programmable prototype for the experimentation of the SDMA concept and use of smart antennas for 
UMTS and GSM/DCS 1800 network. In Proceedings of PIMRC (pp. 534-538). Helsinki, Finland, 1-4 September, 1997.

15. Blogh, J. S., \& Hanzo, L. (2002). Third-generation systems and intelligent wireless networking smart antennas and adaptive modulation. New York, NY: Wiley.

16. Hugl, K., Laurila, J., \& Bonek, E. (1999). Downlink performance of adaptive antenna with null broadening. In Proceedings of IEEE VTC (pp. 872-876). Houston, USA, 4-7 May, 1999.

17. Lo, T. Y. (1972). A mathematical theory of antenna arrays with randomly spaces elements. IEEE Transaction on Antennas and Propagation, 12(3), 129-136.

18. Ochiai, H., Mitran, P., Poor, H. V., \& Tarokh, V. (2005). Collaborative beamforming for distributed wireless ad hoc sensor networks. IEEE Transaction on Signal Processing, 53(11), 4110-4124.

19. Dong, L., Petropulu, A. P., \& Poor, H. V. (2008). A cross-layer approach to collaborative beamforming for wireless ad hoc networks. IEEE Transaction on Signal Processing, 56(7), 2981-2993.

20. Zarifi, K., Affes, S., \& Ghrayeb, A. (2009). Distributed beamforming for wireless sensor networks with random node location. In Proceedings of ICASSP (pp. 2261-2264). Taipei, Taiwan, 19-24 April, 2009.

21. Mudumbai, R., Barriac, G., \& Madhow, U. (2007). On the feasibility of distributed beamforming in wireless sensor networks. IEEE Transaction on Wireless Communication, 6(5), 1754-1763.

22. Brown, D. R., III, \& Poor, H. V. (2008). Time-slotted round-trip carrier synchronization for distributed beamforming. IEEE Transaction on Signal Processing, 56(11), 5630-5643.

23. Bucklew, J. A., \& Sethares, W. A. (2008). Convergence of a class of decentralized beamforming algorithms. IEEE Transaction on Signal Processing, 56(6), 2280-2288.

24. Lian, X., Nikookar, H., \& Ligthart, L. P. (2009). Adaptive OFDM beamformer with constrained weights for cognitive radio. In Proceedings of IEEE VTC Spring (pp. 1-5). Barcelona, Spain, 26-29 April, 2009.

25. Applebaum, S. P., \& Chapman, D. J. (1976). Adaptive array with main beam constrains. IEEE Transactions on Antennas and Propagation, 24(5), 650-662.

26. Applebaum, S. P. (1976). Adaptive arrays. IEEE Transactions on Antennas and Propagation, 24(5), 585-598.

27. Widrow, B., Mantey, P. E., Griffiths, L. J., \& Goode, B. B. (1967). Adaptive antenna systems. Proceedings of IEEE, 55(12), 2143-2159.

28. Frost, O. L. (1972). An algorithm for linearly constrained adaptive array processing. Proceedings of IEEE, 60(8), 926-935.

29. Griffiths, L. J. (1969). A simple adaptive algorithm for real-time processing in antenna arrays. Proceedings of IEEE, 57(10), 696-1704.

30. Gordara, L. C. (1969). Application of antenna arrays to mobile communications, Part II: Beam-forming and Direction-of-Arrival considerations. Proceedings of IEEE, 85(8), 1193-1245.

31. Capon, J. (1969). High-resolution frequency-wavenumber spectrum analysis. Proceedings of IEEE, 57(8), 1408-1418.

32. Reed, I. S., Mallett, J. D., \& Brennan, L. E. (1974). Rapid convergence rate in adaptive arrays. IEEE Transaction on Aerospace and Electronic Systems, 10(6), 853-863.

33. Litva, J. (1996). Digital beamforming in wireless communications. Boston, London: Artech House.

34. Godara, L. C. (1997). Application of antenna arrays to mobile communications, Part I: performance improvement, feasibility, and system considerations. Proceedings of IEEE, 85(7), 1029-1060.

35. Krim, H., \& Viberg, M. (1996). Two decades of array signal processing research. IEEE Signal Processing Magazine, pp. 67-94.

36. Treichler, J. R., \& Agee, B. (1983). A new approach to multipath correction of constant modulus signals. IEEE Transaction on Acoustics, Speech and Signal Processing, 31(2), 459-472.

37. Godard, D. N. (1980). Self-recovering equalization and carrier tracking in a two-dimensional data communication system. IEEE Transaction on Communication, 28(11), 1867-1875.

38. Agee, B. G., Schell, S. V., \& Gardner, W. A. (1990). Spectral self-coherent restoral: A new approach to build adaptive signal extraction using antenna arrays. Proceedings of IEEE, 78(4), 753-767.

39. Wu, Q., Wong, K. M., \& Ho, R. (1994). A fast algorithm for adaptive beamforming of cyclic signals. IEE Proceedings on Radar, Sonar and Navigation, 141(6), 312-318.

40. Henriksson, J. A. (1987). Decision-directed diversity combiners-principles and Simulation results. IEEE Journal on Selected Areas in Communications, 5(3), 515-523.

41. Rappaport, T. S. (1996). Wirelss communications: Principles and practice. New Jersey: Prentice Hall.

42. Bell, K. L., Ephraim, Y., \& Van Trees, H. L. (2000). A Bayesian approach to robust adaptive beamforming. IEEE Transaction on Signal Processing, 48(2), 386-398.

43. Lam, C. J., \& Singer, A. C. (4445). Bayesian beamforming for DOA uncertainty: Theory and implementation. IEEE Transaction on Signal Processing, 54(11), 4435-4445. 
44. Filho, D. Z., Panazio, C. M., Cavalcanti, F. R. P., \& Romano, J. M. T. (2001). On downlink beamforming techniques for TDMA/FDD systems. In Proceedings of Symposio Brasileiro de Telecomunicacoes, Fortaleza, Brazil, 20 December, 2001.

45. Liang, Y. C., \& Chin, F. P. S. (2002). FDD DS-CDMA Downlink beamforming by modifying uplink beamforming weights. In Proceedings of IEEE VTC Fall (pp. 170-174), Vancouver, Canada, 24-28 September, 2002.

46. Liang, Y. C., Chin, F. P. S., \& Liu, K. J. R. (2001). Downlink beamforming for DS-CDMA mobile radio with multimedia services. IEEE Transaction on Communication, 49(7), 1288-1298.

47. Mailloux, R. J. (1998). Covariance matrix augmentation to produce adaptive array pattern troughs. Electronics Letters, 31(10), 771-772.

48. Zatman, M. (1998). Production of adaptive array troughs by dispersion synthesis. Electronics Letters, 31(25), 2141-2142.

49. Guerci, J. R. (1999). Theory and application of covariance matrix tapers for robust adaptive beamforming. IEEE Transaction on Signal Processing, 47(4), 977-985.

50. Song, H. C., Kuperman, W. A., Hodgkiss, W.S., Gerstoft, P., \& Kim, J. S. (2004). Robustness of null broadening against source motion. In Proceedings of Sensor Array and Multichannel Signal Processing Workshop (pp. 470-474), Washington, USA, 4-6 August, 2004.

51. Song, H. C., Kuperman, W. A., Hodgkiss, W. S., Gerstoft, P., \& Kim, J. S. (2003). Null broadening with snapshot-deficient covariance matrices in passive sonar. IEEE Journal of Oceanic Engineering, 28(2), 250-261.

52. Riba, J., Goldberg, J., \& Vazquez, G. (1997). Robust beamforming for interference rejection in mobile communications. IEEE Transaction on Acoustic Speech, Signal Processing, 45(1), 271-275.

53. Hugl, K., Laurila, J., \& Bonek, E. (1999). Downlink performance of adaptive antennas with null broadening. In Proceedings of IEEE 49th VTC (pp. 16-20), Houston, USA, 16-20 May, 1999.

54. Xu, Z., \& Zakharov, Y. (2007). Modified null broadening adaptive beamforming: Constrained optimization approach. Electronics Letters, 43(3), 145-146.

55. Yang, Y., \& Wan, C. (2004). Adaptive beampattern synthesis based on broadening. In Proceedings of IEEE Antennas and Propagation Society International Symposium (pp. 3996-3999), California, USA, 20-25 June, 2004.

56. Adali, T., \& Haykin, S. (2010). Adaptive signal processing: Next generation solutions. New Jersey: Wiley.

57. Stuber, G. L. (1996). Principles of mobile communication. Boston, MA: Kluwer.

58. Sadowsky, J. S., \& Kafedziski, V. (1998). On the correlation and scattering functions of the WSSUS channel for mobile communication. IEEE Transaction on Vehicle Technology, 47(1), $270-282$.

59. Abdi, A., Barger, J. A., \& Kaveh, M. (2002). A parametric model for the distribution of the angle of arrival and the associated correlation function and power spectrum at the mobile station. IEEE Transaction on Vehicle Technology, 51(3), 425-434.

60. Laneman, J. N., Tse, D. N. C., \& Wornell, G. W. (2004). Cooperative diversity in wireless networks: Efficient protocols and outage behavior. IEEE Transaction on Information Theory, 50(12), 3062-3080.

61. Johnston, N., \& Aghvami, H. (2007). Comparing WiMAX and HSPA-A guide to the technology. BT Technology Journal, 25(2), 191-199. 


\section{Author Biographies}
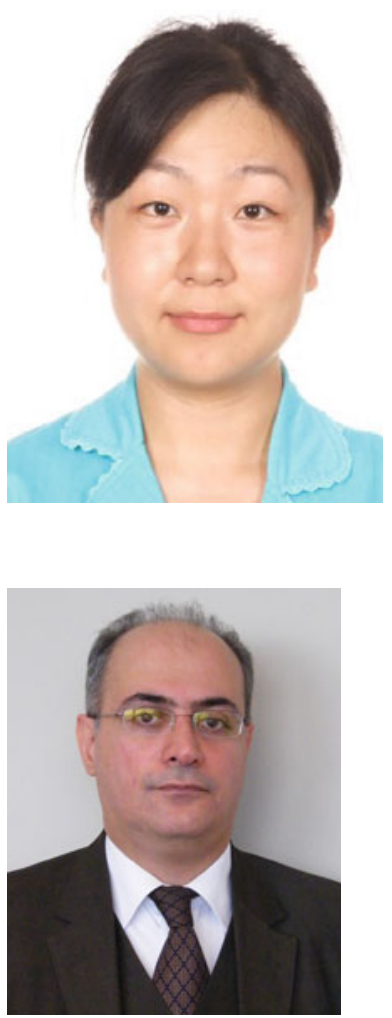

Xiaohua Lian received her B.S. and M.S. degrees in electrical and electronic engineering from Nanjing University of Aeronautics and Astronautics (NUAA), Nanjing, China, in 2002 and 2005, respectively. She is now working towards her Ph.D. in Delft University of Technology in the Nederlands. Her research interests include array signal processing for radar and for wireless communications, smart antenna and cognitive radio, etc.

has published over 100 papers, in refereed international conferences and journals. He is also the recipient of several paper awards of IEEE journals and International Conferences and Symposiums. In 2007 Dr. Nikookar served as the Chair of the $14^{\text {th }}$ IEEE Symposium on Communications and Vehicular Technology (SCVT) in Benelux and in 2008 was the Chairman of the European Wireless Technology Conference (EuWiT) in Amsterdam. He has been technical program committee member of several international conferences and has served as a Guest Editor of several international journals. He is a senior member of the IEEE and the coauthor of the book, Introduction to Ultra Wideband for Wireless Communications, Springer, 2009.

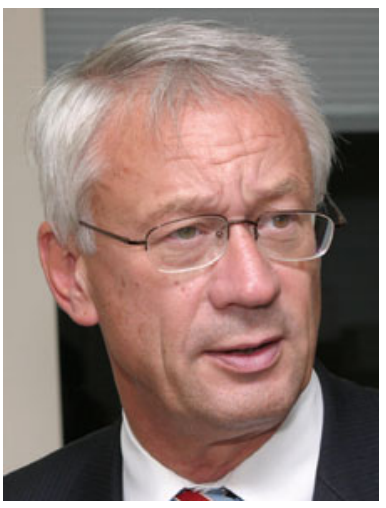

Leo P. Ligthart was born in Rotterdam, the Netherlands, on September 15, 1946. He received an Engineer's degree (cum laude) and a Doctor of Technology degree from Delft University of Technology in 1969 and 1985, respectively. He is fellow of IEE and IEEE. He received Doctorates (honoris causa) at Moscow State Technical University of Civil Aviation in 1999, Tomsk State University of Control Systems and Radioelectronics in 2001 and Military Technical Academy, Romania in 2010. He is academician of the Russian Academy of Transport. Since 1992, he has held the chair of Microwave Transmission, Radar and Remote Sensing in the Faculty of Electrical Engineering, Mathematics and Computer Science, Delft University of Technology. In 1994, he founded the International Research Center for Telecommunications and Radar (IRCTR) and has been the director of IRCTR for more than 16 year. His principal areas of specialization include antennas and propagation, radar and remote sensing, but he has also been active in satellite, mobile and radio communications. He has published over 600 papers and book chapters, and 2 books. 